\title{
Space-Time Estimates on Damped Fractional Wave Equation
}

\author{
Jiecheng Chen, ${ }^{1}$ Dashan Fan, ${ }^{2}$ and Chunjie Zhang $^{3}$ \\ ${ }^{1}$ Department of Mathematics, Zhejiang Normal University, Jinhua 321004, China \\ ${ }^{2}$ Department of Mathematics, University of Wisconsin-Milwaukee, Milwaukee, WI 53201, USA \\ ${ }^{3}$ School of Science, Hangzhou Dianzi University, Hangzhou, Zhejiang 310018, China
}

Correspondence should be addressed to Chunjie Zhang; purezhang@hdu.edu.cn

Received 26 July 2013; Accepted 27 January 2014; Published 4 May 2014

Academic Editor: Nasser-eddine Tatar

Copyright (C) 2014 Jiecheng Chen et al. This is an open access article distributed under the Creative Commons Attribution License, which permits unrestricted use, distribution, and reproduction in any medium, provided the original work is properly cited.

We obtain space-time estimates on the solution $u(t, x)$ to the Cauchy problem of damped fractional wave equation. We mainly focus on the linear equation. The almost everywhere convergence of the solution to linear equations as $t \rightarrow 0^{+}$is also studied, with the initial data satisfying certain regularity conditions.

\section{Introduction}

Let $(t, x) \in[0, \infty) \times \mathbb{R}^{n}, \alpha>0, a, b \in \mathbb{C}$, and let $\Delta$ be the Laplace operator. We consider the following Cauchy problem:

$$
a u_{t t}(t)(x)+2 b u_{t}(t, x)+(-\Delta)^{\alpha} u(t, x)=0,
$$

with initial conditions

$$
u(0, x)=f(x), \quad u_{t}(0, x)=g(x) .
$$

Here, as usual, the fractional Laplacian $(-\Delta)^{\alpha}$ is defined through the Fourier transform:

$$
\widehat{(-\Delta)^{\alpha}} f(\xi)=|\xi|^{2 \alpha} \widehat{f}(\xi)
$$

for all test functions $f$. The partial differential equation in (1) is significantly interesting in mathematics, physics, biology, and many scientific fields. It is the wave equation when $a=$ $1, b=0$, and $\alpha=1$ and it is the half wave equation when $a=0,2 b=i$, and $\alpha=1 / 2$. As known, the wave equation is one of the most fundamental equations in physics. Another fundamental equation in physics is the Schrödinger equation which can be deduced from (1) by letting $a=0,2 b=i$, and $\alpha=1$. The Schrödinger equation plays a remarkable role in the study of quantum mechanics and many other fields in physics. Also, (1) is the heat equation when $a=0, b=1 / 2$, and $\alpha=1$.

As we all know, wave equation, Schrödinger equation, heat equation, and Laplace equations are most important and fundamental types of partial differential equations. The researches on these equations and their related topics are well-mature and very rich and they are still quite active and robust research fields in modern mathematics. The reader is readily to find hundreds and thousands of interesting papers by searching the Google Scholar or checking the MathSciNet in AMS. Here we list only a few of them that are related to this research paper [1-23].

With an extra damping term $2 b u_{t}(t, x)$ in the wave equation, one obtains the damped wave equation

$$
u_{t t}(t, x)+2 b u_{t}(t, x)-\Delta u(t, x)=0, \quad b>0 .
$$

We observe that there are also a lot of research articles in the literature addressing the above damped wave equation. Among numerous research papers we refer to [24-35] and the references therein. From the reference papers, we find that the damped wave equation (4) is well studied in many interesting topics such as the local and global well-posedness of some linear, semilinear, and nonlinear Cauchy problems and asymptotic and regularity estimates of the solution. We observe that the space frames of these studies focus on the Lebesgue spaces and the Lebesgue Sobolev spaces.

These observations motivate us to consider the Cauchy problem of a more general fractional damped wave equation:

$$
\begin{gathered}
u_{t t}(t, x)+2 b u_{t}(t, x)+(-\Delta)^{\alpha} u(t, x)=0, \\
u(0, x)=f(x), \quad u_{t}(0, x)=g(x),
\end{gathered}
$$


where $\alpha, b>0$ are fixed constants. According to our best knowledge, the fractional damped wave equation was not studied in the literature, except the wave case $\alpha=1$. So our plan is to first study the linear equation (5) and to prove some $L^{p} \rightarrow L^{q}$ estimates. In our later works, we will use those estimates to study the well-posedness of certain nonlinear equations. We can easily check that the solution of (5) is formally given by

$$
\begin{aligned}
u_{b} & (f, g)(t, x) \\
& =\left\{e^{-b t} \cosh (t \sqrt{L}) f+e^{-b t} \frac{\sinh (t \sqrt{L})}{\sqrt{L}}(b f+g)\right\},
\end{aligned}
$$

where $L$ is the Fourier multiplier with symbol $b^{2}-|\xi|^{2 \alpha}$ (see Appendix). Thus our interest will focus on the operators

$$
\begin{aligned}
& T_{\alpha, b}(t):=e^{-b t} \cosh (t \sqrt{L}), \\
& S_{\alpha, b}(t):=e^{-b t} \frac{\sinh (t \sqrt{L})}{\sqrt{L}} .
\end{aligned}
$$

Using dilation, we will restrict ourselves to the case $b=1$ so the theorems are all stated for $u(f, g)=u_{1}(f, g)$ (see Remark 6). We now denote

$$
\begin{aligned}
& S_{\alpha}(t)=S_{\alpha, 1}(t)=e^{-t} \frac{\sinh \left(t \sqrt{1-(-\Delta)^{\alpha}}\right)}{\sqrt{1-(-\Delta)^{\alpha}}}, \\
& T_{\alpha}(t)=T_{\alpha, 1}(t)=e^{-t} \cosh \left(t \sqrt{1-(-\Delta)^{\alpha}}\right) .
\end{aligned}
$$

These two operators are both convolution. We denote their kernels by $\Omega_{\alpha}(t)$ and $K_{\alpha}(t)$. Thus, we may write

$$
T_{\alpha}(t) f=K_{\alpha}(t) * f, \quad S_{\alpha}(t) f=\Omega_{\alpha}(t) * f .
$$

To state our main results, we need the following definition of admissible triplet.

Definition 1. A triplet $(p, q, r)$ is called $\sigma$-admissible if

$$
\frac{1}{q} \leq \sigma\left(\frac{1}{r}-\frac{1}{p}\right),
$$

where $0<r \leq p \leq+\infty, r<q<\infty$, and $\sigma>0$. paper.

The following theorems are part of the main results in the

Theorem 2. Let $\alpha>0$ and let $(p, q, r)$ be $n / 2 \alpha$-admissible and $1 \leq p \leq+\infty$. Then for any $\beta>n \alpha|1 / p-1 / 2|$, one has

$$
\begin{aligned}
& \left(\int_{0}^{\infty}\left\|K_{\alpha}(t) * f\right\|_{L^{p}\left(\mathbb{R}^{n}\right)}^{q} d t\right)^{1 / q} \preceq\|f\|_{H^{r}\left(\mathbb{R}^{n}\right)}+\|f\|_{\dot{L}_{\beta}^{p}\left(\mathbb{R}^{n}\right)}, \\
& \left(\int_{0}^{\infty}\left\|\Omega_{\alpha}(t) * f\right\|_{L^{p}\left(\mathbb{R}^{n}\right)}^{q} d t\right)^{1 / q} \preceq\|f\|_{H^{r}\left(\mathbb{R}^{n}\right)}+\|f\|_{\dot{L}_{\beta-\alpha}^{p}\left(\mathbb{R}^{n}\right)} .
\end{aligned}
$$

Here, $\dot{L}_{\gamma}^{p}\left(\mathbb{R}^{n}\right)$ denotes the homogeneous Sobolev $L^{p}$ space with order $\gamma$, and $H^{r}$ denotes the real Hardy space.
Theorem 3. Let $\alpha=1,(p, q, r)$ be $n / 2$-admissible and $1 \leq p \leq$ $+\infty$. Then the damped wave operators satisfy

$$
\begin{aligned}
& \left(\int_{0}^{\infty}\left\|K_{1}(t) * f\right\|_{L^{p}\left(\mathbb{R}^{n}\right)}^{q} d t\right)^{1 / q} \preceq\|f\|_{H^{r}\left(\mathbb{R}^{n}\right)}+\|f\|_{\dot{L}^{p}\left(\mathbb{R}^{n}\right)}, \\
& \left(\int_{0}^{\infty}\left\|\Omega_{1}(t) * f\right\|_{L^{p}\left(\mathbb{R}^{n}\right)}^{q} d t\right)^{1 / q} \preceq\|f\|_{H^{r}\left(\mathbb{R}^{n}\right)}+\|f\|_{\dot{L}_{\beta-1}^{p}}\left(\mathbb{R}^{n}\right)
\end{aligned}
$$

for any $\beta>(n-1)|1 / p-1 / 2|$.

By the above theorems, we easily obtain the following space-time estimates on the solution $u(t, x)$.

Theorem 4. Let $\alpha>0$ and let $(p, q, r)$ be $n / 2 \alpha$-admissible and $1 \leq p \leq+\infty$. For the solution $u(t, x)$ of $(5)$, one has

$$
\begin{aligned}
& \left(\int_{0}^{\infty}\|u\|_{L^{p}\left(\mathbb{R}^{n}\right)}^{q} d t\right)^{1 / q} \preceq\|f\|_{H^{r}\left(\mathbb{R}^{n}\right)}+\|f\|_{\dot{L}_{\beta}^{p}\left(\mathbb{R}^{n}\right)}+\|f\|_{\dot{L}_{\beta-\alpha}^{p}} \\
& +\|g\|_{H^{r}\left(\mathbb{R}^{n}\right)}+\|g\|_{\dot{L}_{\beta-\alpha}^{p}\left(\mathbb{R}^{n}\right)} .
\end{aligned}
$$

Theorem 5. Let $\alpha=1,(p, q, r)$ be $n / 2$-admissible and $1 \leq p \leq$ $+\infty$. The solution $u(t, x)$ of the damped wave equation satisfies

$$
\begin{aligned}
\left(\int_{0}^{\infty}\|u\|_{L^{p}\left(\mathbb{R}^{n}\right)}^{q} d t\right)^{1 / q} \preceq & \|f\|_{H^{r}\left(\mathbb{R}^{n}\right)}+\|f\|_{\dot{L}_{\beta}^{p}\left(\mathbb{R}^{n}\right)}+\|f\|_{\dot{L}_{\beta-1}^{p}} \\
& +\|g\|_{H^{r}\left(\mathbb{R}^{n}\right)}+\|g\|_{\dot{L}_{\beta-1}^{p}\left(\mathbb{R}^{n}\right)},
\end{aligned}
$$

for any $\beta>(n-1)|1 / p-1 / 2|$.

Remark 6. For (5) with general $b>0$, it is not hard to see that

$$
\begin{aligned}
& T_{a, b}(t) f(x)=T_{a, 1}(s) f_{b}\left(b^{1 / \alpha} x\right), \\
& S_{a, b}(t) f(x)=S_{a, 1}(s)\left(\frac{1}{b} f_{b}\right)\left(b^{1 / \alpha} x\right),
\end{aligned}
$$

where $s=b t$ and $f_{b}(x)=f\left(b^{-1 / \alpha} x\right)$. Therefore,

$$
u_{b}(f, g)(t, x)=u\left(f_{b}, \frac{1}{b} g_{b}\right)\left(b t, b^{1 / \alpha} x\right)
$$

and by applying Theorem 4, we have

$$
\begin{aligned}
& \left(\int_{0}^{\infty}\left\|u_{b}\right\|_{L^{p}}^{q} d t\right)^{1 / q} \\
& \quad \leq b^{(n / \alpha)(1 / r-1 / p)-1 / q}\left(\|f\|_{H^{r}}+b^{-1}\|g\|_{H^{r}}\right) \\
& \quad+b^{-\beta / \alpha-1 / q}\left(\|f\|_{\dot{L}_{\beta}^{p}}+b\|f\|_{\dot{L}_{\beta-\alpha}^{p}}+\|g\|_{\dot{L}_{\beta-\alpha}^{p}}\right) .
\end{aligned}
$$

For $\alpha=1$, we have a similar result using Theorem 5 .

In the statement of these theorems, the notation $A \preceq$ $B$ means that there is a constant $C>0$ independent of 
all essential variables such that $A \leq C B$. Also, throughout this paper, we use the notation $A \simeq B$ to mean that there exist positive constants $C$ and $c$, independent of all essential variables such that

$$
c B \leq A \leq C B .
$$

It is easy to see that, by the linearity, we only need to prove Theorems 2 and 3. To this end, we will carefully study the kernels

$$
\begin{aligned}
& K_{\alpha}(t)(x)=e^{-t} \int_{\mathbb{R}^{n}} \cosh \left(t \sqrt{1-|\xi|^{2 \alpha}}\right) e^{i\langle x, \xi\rangle} d \xi, \\
& \Omega_{\alpha}(t)(x)=e^{-t} \int_{\mathbb{R}^{n}} \frac{\sinh \left(t \sqrt{1-|\xi|^{2 \alpha}}\right)}{\sqrt{1-|\xi|^{2 \alpha}}} e^{i\langle x, \xi\rangle} d \xi .
\end{aligned}
$$

Using the linearization

$$
(1+s)^{1 / 2} \simeq 1+\frac{s}{2}
$$

for small $|\xi|$, we have

$$
\begin{aligned}
\cosh \left(t \sqrt{1-|\xi|^{2 \alpha}}\right) & \simeq \cosh \left(t\left(1-\frac{|\xi|^{2 \alpha}}{2}\right)\right) \\
& =\frac{e^{t\left(1-|\xi|^{2 \alpha} / 2\right)}+e^{-t\left(1-|\xi|^{2 \alpha} / 2\right)}}{2} .
\end{aligned}
$$

Thus for small $|\xi|$,

$$
\begin{aligned}
& e^{-t} \cosh \left(t \sqrt{1-|\xi|^{2 \alpha}}\right) \\
& \simeq \frac{e^{-t} e^{t\left(1-|\xi|^{2 \alpha} / 2\right)}+e^{-t} e^{-t\left(1-|\xi|^{2 \alpha} / 2\right)}}{2} \simeq e^{-t|\xi|^{2 \alpha} / 2} .
\end{aligned}
$$

This indicates that, for $|\xi|$ near zero, $T_{\alpha}$ behaves like the fractional heat operator (see $[11,29,30,36,37])$.

For large $|\xi|$, we similarly have

$$
\begin{aligned}
\cosh \left(t \sqrt{1-|\xi|^{2 \alpha}}\right) & =\cosh \left(i t|\xi|^{\alpha} \sqrt{\left(1-|\xi|^{-2 \alpha}\right)}\right) \\
& \simeq \frac{e^{i t|\xi|^{\alpha}}-e^{-i t|\xi|^{\alpha}}}{2} .
\end{aligned}
$$

This indicates that as $|\xi|$ near $\infty, e^{t} T_{\alpha}$ behaves like the wave operator if $\alpha=1$ and like the Schrödinger operator if $\alpha=2$; see $[12,16,38,39]$.

In the same manner, the operator $S_{\alpha}(t)$ behaves the same as the operator $T_{\alpha}$. Based on these facts, we will estimate the kernels in their low frequencies, median frequencies, and high frequencies, separately, by using different methods. We will estimate the kernels in Section 2 and complete the proofs of main theorems in Section 3. Finally, in Section 4, we will study the almost everywhere convergence for the solution $u(t, x)$ as $t \rightarrow 0^{+}$. The similar convergence theorem for Schrödinger operator $e^{i t \Delta} f(x)$ has been widely studied; see $[3,40-44]$.

\section{Estimates on Kernels}

As we mentioned in the first section, we will estimate the kernels $K_{\alpha}(t)$ and $\Omega_{\alpha}(t)$ based on their different frequencies. So we will divide this section into several subsections.

2.1. Estimate for $|\xi|$ near Zero. Let $\phi_{1}$ be a $C^{\infty}$ radial function with support in $\left\{\xi \in \mathbb{R}^{n}:|\xi|^{2 \alpha} \leq 1 / 2\right\}$ and satisfy $\phi_{1} \equiv 1$ whenever $|\xi|^{2 \alpha} \leq 1 / 3$. In this section we are going to obtain the decay estimates on the kernels

$$
\begin{aligned}
& K_{\alpha, 0}(t)(x)=e^{-t} \int_{\mathbb{R}^{n}} \phi_{1}(\xi) \cosh \left(t \sqrt{1-|\xi|^{2 \alpha}}\right) e^{i\langle\xi, x\rangle} d \xi, \\
& \Omega_{\alpha, 0}(t)(x)=e^{-t} \int_{\mathbb{R}^{n}} \phi_{1}(\xi) \frac{\sinh \left(t \sqrt{1-|\xi|^{2 \alpha}}\right)}{\sqrt{1-|\xi|^{2 \alpha}}} e^{i\langle\xi, x\rangle} d \xi .
\end{aligned}
$$

With those decay estimates, we then are able to obtain two $H^{p}$ bounds for the convolutions with the above two kernels. Without loss of generality, we assume $0<2 \alpha<1$. This assumption is not essential by tracking the following proofs.

Proposition 7. Let $K_{\alpha, 0}$ and $\Omega_{\alpha, 0}$ be defined as above. For all $t>0$, one has

$$
\begin{aligned}
& \left|K_{\alpha, 0}(t)(x)\right| \preceq(1+t)^{-n / 2 \alpha}\left(1+(1+t)^{-1 / 2 \alpha}|x|\right)^{-n-2 \alpha}, \\
& \left|\Omega_{\alpha, 0}(t)(x)\right| \preceq(1+t)^{-n / 2 \alpha}\left(1+(1+t)^{-1 / 2 \alpha}|x|\right)^{-n-2 \alpha} .
\end{aligned}
$$

Proof. The estimates of two inequalities are the same, so we will prove the first one only.

(i) If $(1+t)^{-1 / 2 \alpha}|x| \leq 1$ and $0<t \leq 1$, then it is obvious to see

$$
\left|K_{\alpha, 0}(t)(x)\right| \preceq \int_{\mathbb{R}^{n}}\left|\phi_{1}(\xi)\right| d \xi \preceq 1 .
$$

(ii) If $(1+t)^{-1 / 2 \alpha}|x| \leq 1$ and $t>1$, then by scaling

$$
\begin{aligned}
& t^{n / 2 \alpha} K_{\alpha, 0}(t)\left(t^{1 / 2 \alpha} x\right) \\
& =t^{n / 2 \alpha} e^{-t} \int_{\mathbb{R}^{n}} \phi_{1}(\xi) \cosh \left(t \sqrt{1-|\xi|^{2 \alpha}}\right) e^{i\left\langle\xi, t^{1 / 2 \alpha} x\right\rangle} d \xi \\
& =e^{-t} \int_{\mathbb{R}^{n}} \phi_{1}\left(t^{-1 / 2 \alpha} \xi\right) \cosh \left(\sqrt{t^{2}-t|\xi|^{2 \alpha}}\right) e^{i\langle\xi, x\rangle} d \xi .
\end{aligned}
$$

Since

$$
\begin{aligned}
& e^{-t} \cosh \left(\sqrt{t^{2}-t|\xi|^{2 \alpha}}\right) \\
& \leq e^{-t} e^{\sqrt{t^{2}-t|\xi|^{2 \alpha}}} \preceq \exp \left(-\frac{|\xi|^{2 \alpha}}{1+\sqrt{1-|\xi|^{2 \alpha} / t}}\right) \\
& \leq e^{-|\xi|^{2 \alpha} / 2},
\end{aligned}
$$


we have

$$
\left|t^{n / 2 \alpha} K_{\alpha, 0}(t)\left(t^{1 / 2 \alpha} x\right)\right| \preceq \int_{\mathbb{R}^{n}}\left|\phi_{1}\left(t^{-1 / 2 \alpha} \xi\right)\right| e^{-|\xi|^{2 \alpha} / 2} d \xi \preceq 1 .
$$

(iii) If $(1+t)^{-1 / 2 \alpha}|x|>1$ and $t>1$, then by (ii) we know

$$
\begin{aligned}
& t^{n / 2 \alpha} K_{\alpha, 0}(t)\left(t^{1 / 2 \alpha} x\right) \\
& \quad=e^{-t} \int_{\mathbb{R}^{n}} \phi_{1}\left(t^{-1 / 2 \alpha} \xi\right) \cosh \left(\sqrt{t^{2}-t|\xi|^{2 \alpha}}\right) e^{i\langle\xi, x\rangle} d \xi .
\end{aligned}
$$

Using the Leibniz rule, we have

$$
\begin{aligned}
& \partial_{\xi_{i}}^{n}\left(\phi_{2}\left(t^{-1 / 2 \alpha} \xi\right) \cosh \left(\sqrt{t^{2}-t|\xi|^{2 \alpha}}\right)\right) \\
& \quad=\sum_{k=0}^{n} C_{n}^{k} \partial_{\xi_{i}}^{n-k}\left(\phi_{2}\left(t^{-1 / 2 \alpha} \xi\right)\right) \partial_{\xi_{i}}^{k}\left(\cosh \left(\sqrt{t^{2}-t|\xi|^{2 \alpha}}\right)\right) .
\end{aligned}
$$

Observe that

$$
\partial_{\xi_{i}}\left(\cosh \left(\sqrt{t^{2}-t|\xi|^{2 \alpha}}\right)\right) \simeq \frac{\sinh \left(\sqrt{t^{2}-t|\xi|^{2 \alpha}}\right)}{\sqrt{t^{2}-t|\xi|^{2 \alpha}}} t|\xi|^{2 \alpha-2} \xi_{i}
$$

For $k \geq 1$, using an induction argument we have

$$
\partial_{\xi_{i}}^{k}\left(\cosh \left(\sqrt{t^{2}-t|\xi|^{2 \alpha}}\right)\right) \simeq F(t, \xi) \sum_{j=1}^{k} \frac{t^{j} \psi_{j}^{k}(\xi)}{\left(\sqrt{t^{2}-t|\xi|^{2 \alpha}}\right)^{j+\epsilon_{k}}}
$$

where $\epsilon_{k} \geq 0, \psi_{j}^{k}(\xi) \preceq|\xi|^{2 j \alpha-k}$, and

$$
F(t, \xi)=\cosh \left(\sqrt{t^{2}-t|\xi|^{2 \alpha}}\right) \quad \text { or } \quad \sinh \left(\sqrt{t^{2}-t|\xi|^{2 \alpha}}\right) \text {. }
$$

For each fixed $x \in \mathbb{R}^{n}$, there exists at least one variable $x_{i}$ such that $\left|x_{i}\right| \geq|x| / n$. By integration by parts $n$ times on the variable $\xi_{i}$, we obtain

$$
\begin{aligned}
& t^{n / 2 \alpha} K_{\alpha, 0}(t)\left(t^{1 / 2 \alpha} x\right) \\
& \simeq \frac{1}{x_{i}^{n}} \sum_{k=0}^{n} C_{n}^{k} e^{-t} \int_{\mathbb{R}^{n}} \partial_{\xi_{i}}^{n-k}\left(\phi_{1}\left(t^{-1 / 2 \alpha} \xi\right)\right) \partial_{\xi_{i}}^{k} \\
& \quad \times\left(\cosh \left(\sqrt{t^{2}-t|\xi|^{2 \alpha}}\right)\right) e^{i\langle\xi, x\rangle} d \xi
\end{aligned}
$$

$$
\begin{aligned}
& \simeq \frac{1}{x_{i}^{n}} \sum_{k=1}^{n-1} \sum_{j=1}^{k} C_{n}^{k} e^{-t} \\
& \quad \times \int_{\mathbb{R}^{n}} \partial_{\xi_{i}}^{n-k}\left(\phi_{1}\left(t^{-1 / 2 \alpha} \xi\right)\right) F(t, \xi)
\end{aligned}
$$$$
\times \frac{t^{j} \psi_{j}^{k}(\xi)}{\left(\sqrt{t^{2}-t|\xi|^{2 \alpha}}\right)^{j+\epsilon_{k}}} e^{i\langle\xi, x\rangle} d \xi
$$$$
+\frac{1}{x_{i}^{n}} \sum_{j=1}^{n} e^{-t} \int_{\mathbb{R}^{n}} \phi_{1}\left(t^{-1 / 2 \alpha} \xi\right) F(t, \xi)
$$$$
\times \frac{t^{j} \psi_{j}^{n}(\xi)}{\left(\sqrt{t^{2}-t|\xi|^{2 \alpha}}\right)^{j+\epsilon_{k}}} e^{i\langle\xi, x\rangle} d \xi
$$$$
+\frac{1}{x_{i}^{n}} e^{-t} \int_{\mathbb{R}^{n}} \partial_{\xi_{i}}^{n}\left(\phi_{1}\left(t^{-1 / 2 \alpha} \xi\right)\right)
$$$$
\times \cosh \left(\sqrt{t^{2}-t|\xi|^{2 \alpha}}\right) e^{i\langle\xi, x\rangle} d \xi .
$$

The main terms needed to be estimated are

$$
\frac{1}{x_{i}^{n}} e^{-t} \int_{\mathbb{R}^{n}} \phi_{1}\left(t^{-1 / 2 \alpha} \xi\right) F(t, \xi) \frac{t^{j} \psi_{j}^{n}(\xi)}{\left(\sqrt{t^{2}-t|\xi|^{2 \alpha}}\right)^{j+\epsilon_{k}}} e^{i\langle\xi, x\rangle} d \xi,
$$

with $j=1,2, \ldots, n$. The other terms can be treated easily by further taking integration by parts.

We let $\Phi$ be a $C^{\infty}$ radial function satisfying $\Phi(\xi)=1$ if $|\xi| \leq 1$ and $\Phi(\xi)=0$ if $|\xi|>2$. Let $\Psi(\xi)=1-\Phi(\xi)$. By the partition of unity we write

$$
\begin{gathered}
\frac{1}{x_{i}^{n}} e^{-t} \int_{\mathbb{R}^{n}} \phi_{1}\left(t^{-1 / 2 \alpha} \xi\right) F(t, \xi) \frac{t^{j} \psi_{j}^{n}(\xi)}{\left(\sqrt{t^{2}-t|\xi|^{2 \alpha}}\right)^{j+\epsilon_{k}}} e^{i\langle\xi, x\rangle} d \xi \\
=\frac{1}{x_{i}^{n}} e^{-t} \int_{\mathbb{R}^{n}} \phi_{1}\left(t^{-1 / 2 \alpha} \xi\right) \Phi(|x| \xi) F(t, \xi)
\end{gathered}
$$

$$
\begin{array}{r}
\times \frac{t^{j} \psi_{j}^{n}(\xi)}{\left(\sqrt{t^{2}-t|\xi|^{2 \alpha}}\right)^{j+\epsilon_{k}}} e^{i\langle\xi, x\rangle} d \xi \\
+\frac{1}{x_{i}^{n}} e^{-t} \int_{\mathbb{R}^{n}} \phi_{1}\left(t^{-1 / 2 \alpha} \xi\right) \Psi(|x| \xi) F(t, \xi) \\
\times \frac{t^{j} \psi_{j}^{n}(\xi)}{\left(\sqrt{t^{2}-t|\xi|^{2 \alpha}}\right)^{j+\epsilon_{k}}} e^{i\langle\xi, x\rangle} d \xi
\end{array}
$$

$=I_{1}+I_{2}$. 
We note that $t>1$, and the support of $\phi_{1}\left(t^{-1 / 2 \alpha} \xi\right)$ together with (28) implies

$$
\begin{aligned}
e^{-t} F(t, \xi) \frac{t^{j}}{\left(\sqrt{t^{2}-t|\xi|^{2 \alpha}}\right)^{j+\epsilon_{k}}} \\
\preceq e^{-t} e^{\sqrt{t^{2}-t|\xi|^{2 \alpha}}} \preceq \exp \left(-\frac{|\xi|^{2 \alpha}}{2}\right) .
\end{aligned}
$$

Therefore

$$
\begin{aligned}
\left|I_{1}\right| & \preceq \frac{1}{|x|^{n}} \int_{\mathbb{R}^{n}} e^{-|\xi|^{2 \alpha} / 2}\left|\phi_{1}\left(t^{-1 / 2 \alpha \xi}\right) \Phi(|x| \xi)\right||\xi|^{2 j \alpha-n} d \xi \\
& \preceq \frac{1}{|x|^{n}} \int_{|\xi| \leq 2 /|x|}|\xi|^{2 j \alpha-n} d \xi \\
& \preceq|x|^{-n-2 j \alpha} .
\end{aligned}
$$

By integration by parts,

$$
\begin{aligned}
& \left|I_{2}\right| \\
& \preceq \frac{1}{|x|^{n+1}} e^{-t} \\
& \times \mid \int_{\mathbb{R}^{n}} \Psi(|x| \xi) \\
& \times \partial_{\xi_{i}}\left(\phi_{1}\left(t^{-1 / 2 \alpha} \xi\right) F(t, \xi) \frac{t^{j} \psi_{j}^{n}(\xi)}{\left(\sqrt{t^{2}-t|\xi|^{2 \alpha}}\right)^{j+\epsilon_{k}}}\right) e^{i\langle\xi, x\rangle} d \xi \\
& +\frac{1}{|x|^{n+1}} e^{-t} \\
& \times \mid \int_{\mathbb{R}^{n}} \partial_{\xi_{i}}(\Psi(|x| \xi)) \phi_{1}\left(t^{-1 / 2 \alpha} \xi\right) F(t, \xi) \\
& \times \frac{t^{j} \psi_{j}^{n}(\xi)}{\left(\sqrt{t^{2}-t|\xi|^{2 \alpha}}\right)^{j+\epsilon_{k}}} e^{i\langle\xi, x\rangle} d \xi \\
& =J_{1}+J_{2} \text {. }
\end{aligned}
$$

Here, an easy computation gives

$$
\begin{aligned}
J_{1} & \preceq \frac{1}{|x|^{n+1}} \int_{|\xi| \geq 1 /|x|}|\Psi(|x| \xi)| e^{-|\xi|^{2 \alpha} / 2}|\xi|^{2 j \alpha-n-1} d \xi \\
& \leq \min \left\{\frac{1}{|x|^{n+1}}, \frac{1}{|x|^{n+2 \alpha}}\right\} .
\end{aligned}
$$

For $J_{2}$, noting that

$$
\left|\partial_{\xi_{i}}(\Psi(|x| \xi))\right| \preceq|x| \Psi^{\prime}(|x| \xi)
$$

and $\Psi^{\prime}(s)$ is supported in $[1 / 2,2]$, we have

$$
J_{2} \preceq \frac{1}{|x|^{n}} \int_{1 / 2|x| \leq|\xi| \leq 2 /|x|}|\xi|^{2 j \alpha-n} d \xi \preceq \frac{1}{|x|^{n+2 j \alpha}}
$$

(iv) If $(1+t)^{-1 / 2 \alpha}|x|>1$ and $0<t \leq 1$, then a similar argument, without scaling, shows that

$$
\left|K_{\alpha, 0}(t)(x)\right| \preceq \frac{1}{|x|^{n+2 \alpha}} .
$$

The proposition now follows from (i)-(iv).

Proposition 8. Let $f \in H^{r}\left(\mathbb{R}^{n}\right)$. Then for any $t>0$ and $0<$ $r \leq p<+\infty$,

$$
\begin{aligned}
& \left\|K_{\alpha, 0}(t) * f\right\|_{H^{p}\left(\mathbb{R}^{n}\right)} \preceq(1+t)^{-(n / 2 \alpha)(1 / r-1 / p)}\|f\|_{H^{r}\left(\mathbb{R}^{n}\right)}, \\
& \left\|\Omega_{\alpha, 0}(t) * f\right\|_{H^{p}\left(\mathbb{R}^{n}\right)} \preceq(1+t)^{-(n / 2 \alpha)(1 / r-1 / p)}\|f\|_{H^{r}\left(\mathbb{R}^{n}\right)} .
\end{aligned}
$$

Particularly, we have

$$
\begin{aligned}
& \left\|K_{\alpha, 0}(t) * f\right\|_{L^{\infty}\left(\mathbb{R}^{n}\right)} \preceq(1+t)^{-n / 2 \alpha r}\|f\|_{H^{r}\left(\mathbb{R}^{n}\right)}, \\
& \left\|\Omega_{\alpha, 0}(t) * f\right\|_{L^{\infty}\left(\mathbb{R}^{n}\right)} \preceq(1+t)^{-n / 2 \alpha r}\|f\|_{H^{r}\left(\mathbb{R}^{n}\right)} .
\end{aligned}
$$

Proof. We prove the proposition for the kernel $K_{\alpha, 0}$ only, since the proof for the other one is exactly the same. Let us first consider the case $p=+\infty$ and $0<r<1$. Invoking an interpolation argument $[45,46]$, we may assume that $n(1 / r-$ $1)$ is a positive integer. Thus the dual space of $H^{r}$ is the homogeneous Lipschitz space $\dot{\Lambda}_{n(1 / r-1)}\left(\mathbb{R}^{n}\right)$ (one can see the definition in [46]), which is exactly the homogeneous Hölder space $\dot{C}^{n(1 / r-1)}\left(\mathbb{R}^{n}\right)$. By duality we have

$$
\left\|K_{\alpha, 0}(t) * f\right\|_{L^{\infty}} \preceq \sup _{x \in \mathbb{R}^{n}}\left\|K_{\alpha, 0}(t)(x-\cdot)\right\|_{\dot{C}^{n(1 / r-1)}}\|f\|_{H^{r}} .
$$

If $t \geq 1$, it is easy to check that

$$
\begin{aligned}
& \sup _{x \in \mathbb{R}^{n}}\left\|K_{\alpha, 0}(t)(x-\cdot)\right\|_{\dot{C}^{n(1 / r-1)}} \\
& =\sup _{x \in \mathbb{R}^{n}}\left\|K_{\alpha, 0}(t)\left(t^{1 / 2 \alpha} x-\cdot\right)\right\|_{\dot{C}^{n(1 / r-1)}} \\
& \leq t^{-n / 2 \alpha r} \sup _{x \in \mathbb{R}^{n}} \mid \int_{\mathbb{R}^{n}} \phi_{1}\left(t^{-1 / 2 \alpha} \xi\right) P(\xi) e^{-t} \\
& \quad \times \cosh \left(\sqrt{t^{2}-t|\xi|^{2 \alpha}}\right) e^{i\langle\xi, x\rangle} d \xi \mid,
\end{aligned}
$$

where $P(\xi)$ is a homogeneous polynomial of degree $n(1 / r-1)$. Thus, using the same argument as before we obtain

$$
\left\|K_{\alpha, 0}(t) * f\right\|_{L^{\infty}} \preceq t^{-n / 2 \alpha r}\|f\|_{H^{r}}
$$


If $0<t \leq 1$,

$$
\begin{aligned}
& \sup _{x \in \mathbb{R}^{n}}\left\|K_{\alpha, 0}(t)(x-\cdot)\right\|_{\dot{C}^{n(1 / r-1)}} \\
& \quad \preceq \sup _{x \in \mathbb{R}^{n}}\left|\int_{\mathbb{R}^{n}} \phi_{1}(\xi) P(\xi) e^{-t} \cosh \left(t \sqrt{1-|\xi|^{2 \alpha}}\right) e^{i\langle\xi, x\rangle} d \xi\right| \\
& \quad \preceq 1 .
\end{aligned}
$$

This shows that, for all $0<r<1$,

$$
\left\|K_{\alpha, 0}(t) * f\right\|_{L^{\infty}} \preceq(1+t)^{-n / 2 \alpha r}\|f\|_{H^{r}} .
$$

On the other hand, if we write

$$
m(t, \xi)=\widehat{K_{\alpha, 0}(t)}(\xi)=e^{-t} \phi_{1}(\xi) \cosh \left(t \sqrt{1-|\xi|^{2 \alpha}}\right),
$$

then by checking the proof of Proposition 7, we find

$$
\left|\partial_{\xi}^{k} m(t, \xi)\right| \preceq|\xi|^{-|k|}
$$

for all multi-indices $k$. So by the Calderón-Torchinsky multiplier theorem [47], we also have, for all $0<r<1$,

$$
\left\|K_{\alpha, 0}(t) * f\right\|_{H^{r}} \preceq\|f\|_{H^{r}} .
$$

Now interpolating between (51) and (54), we finish the proof for $0<r<1$.

For the case $1 \leq r \leq+\infty$, we use Young's inequality to get

$$
\left\|K_{\alpha, 0} *(t) f\right\|_{L^{p}}=\left\|K_{\alpha, 0}(t)\right\|_{L^{q}}\|f\|_{L^{r}}
$$

where $1 / r+1 / q=1 / p+1$. By Proposition 7 ,

$$
\begin{aligned}
& \left\|K_{\alpha, 0}(t)\right\|_{L^{q}} \\
& \quad \leq\left(\int_{\mathbb{R}^{n}}\left|(1+t)^{-n / 2 \alpha}\left(1+(1+t)^{-1 / 2 \alpha}|x|\right)^{-n-2 \alpha}\right|^{q} d x\right)^{1 / q} \\
& \quad \leq(1+t)^{-(n / 2 \alpha)(1 / r-1 / p)} .
\end{aligned}
$$

2.2. Estimate for $|\xi|$ Lying in the Mid-Interval. Let $\phi_{2}$ be a $C^{\infty}$ radial function with support in $\left\{\xi \in \mathbb{R}^{n}: 1 / 4 \leq|\xi|^{2 \alpha} \leq 200\right\}$ and satisfy $\phi_{1} \equiv 1$ whenever $1 / 3 \leq|\xi|^{2 \alpha} \leq 100$. We first will obtain the decay estimate on the kernels

$$
\begin{aligned}
& K_{\alpha, m}(t)(x)=e^{-t} \int_{\mathbb{R}^{n}} \phi_{2}(\xi) \cosh \left(t \sqrt{1-|\xi|^{2 \alpha}}\right) e^{i\langle\xi, x\rangle} d \xi \\
& \Omega_{\alpha, m}(t)(x)=e^{-t} \int_{\mathbb{R}^{n}} \phi_{2}(\xi) \frac{\sinh \left(t \sqrt{1-|\xi|^{2 \alpha}}\right)}{\sqrt{1-|\xi|^{2 \alpha}}} e^{i\langle\xi, x\rangle} d \xi
\end{aligned}
$$

and then prove the mapping properties of the convolution operators with the above kernels. As in Section 2.1, we assume $0<2 \alpha<1$ without loss of generality.
Proposition 9. For all $t>0$ and $N>0$, we have

$$
\begin{aligned}
& \left|K_{\alpha, m}(t)(x)\right| \preceq(1+t)^{-n / 2 \alpha-N}\left(1+(1+t)^{-1 / 2 \alpha}|x|\right)^{-n-2 \alpha}, \\
& \left|\Omega_{\alpha, m}(t)(x)\right| \preceq(1+t)^{-n / 2 \alpha-N}\left(1+(1+t)^{-1 / 2 \alpha}|x|\right)^{-n-2 \alpha} .
\end{aligned}
$$

Proof. If $(1+t)^{-1 / 2 \alpha}|x| \leq 1$, then the proof is the same as (i) and (ii) in the proof of Proposition 7. So we assume $(1+$ $t)^{-1 / 2 \alpha}|x|>1$ and $t>1$. In the case of $t \leq 1$, we use the same proof as the following argument for $t>1$, without taking the scaling kernel.

For $t>1$, consider the scaling kernel

$$
\begin{aligned}
& t^{n / 2 \alpha} K_{\alpha, m}(t)\left(t^{1 / 2 \alpha} x\right) \\
& =t^{n / 2 \alpha} e^{-t} \int_{\mathbb{R}^{n}} \phi_{2}(\xi) \cosh \left(t \sqrt{1-|\xi|^{2 \alpha}}\right) e^{i\left\langle\xi, t^{1 / 2 \alpha} x\right\rangle} d \xi \\
& =e^{-t} \int_{\mathbb{R}^{n}} \phi_{2}\left(t^{-1 / 2 \alpha} \xi\right) \cosh \left(\sqrt{t^{2}-t|\xi|^{2 \alpha}}\right) e^{i\langle\xi, x\rangle} d \xi .
\end{aligned}
$$

By the Leibniz rule,

$$
\begin{aligned}
& \partial_{\xi_{i}}^{n}\left(\phi_{2}\left(t^{-1 / 2 \alpha} \xi\right) \cosh \left(\sqrt{t^{2}-t|\xi|^{2 \alpha}}\right)\right) \\
& \quad=\sum_{k=0}^{n} C_{n}^{k} \partial_{\xi_{i}}^{n-k}\left(\phi_{2}\left(t^{-1 / 2 \alpha} \xi\right)\right) \partial_{\xi_{i}}^{k}\left(\cosh \left(\sqrt{t^{2}-t|\xi|^{2 \alpha}}\right)\right) .
\end{aligned}
$$

Next we prove the following estimate:

$$
\begin{aligned}
& \left|\partial_{\xi_{i}}^{k}\left(\cosh \left(\sqrt{t^{2}-t|\xi|^{2 \alpha}}\right)\right)\right| \\
& \quad \leq \exp \left(\left|\sqrt{t^{2}-t|\xi|^{2 \alpha}}\right|\right) \sum_{j=1}^{k} t^{j}|\xi|^{2 j \alpha-k} .
\end{aligned}
$$

In fact, using Taylor's expansion, we have

$$
\cosh \left(\sqrt{t^{2}-t|\xi|^{2 \alpha}}\right)=\sum_{l=0}^{+\infty} \frac{\left(t^{2}-t|\xi|^{2 \alpha}\right)^{l}}{(2 l) !} .
$$

Then by an easy computation,

$$
\begin{aligned}
& \partial_{\xi_{i}}\left(\cosh \left(\sqrt{t^{2}-t|\xi|^{2 \alpha}}\right)\right) \simeq \sum_{l=1}^{+\infty} \frac{l\left(t^{2}-t|\xi|^{2 \alpha}\right)^{l-1}}{(2 l) !} t|\xi|^{2 \alpha-2} \xi_{i} \\
& \partial_{\xi_{i}}^{2}\left(\cosh \left(\sqrt{t^{2}-t|\xi|^{2 \alpha}}\right)\right) \\
& \simeq \sum_{l=2}^{+\infty} \frac{l(l-1)\left(t^{2}-t|\xi|^{2 \alpha}\right)^{l-2}}{(2 l) !} t^{2}|\xi|^{4 \alpha-4} \xi_{i}^{2} \\
& +\sum_{l=1}^{+\infty} \frac{\left(\left(t^{2}-t|\xi|^{2 \alpha}\right)^{l-1}\right.}{(2 l) !} t\left(|\xi|^{2 \alpha-4} \xi_{i}^{2}+|\xi|^{2 \alpha-2}\right) .
\end{aligned}
$$


Thus, by the induction, we have

$$
\begin{aligned}
& \partial_{\xi_{i}}^{k}\left(\cosh \left(\sqrt{t^{2}-t|\xi|^{2 \alpha}}\right)\right) \\
& \simeq \sum_{j=1}^{k} \sum_{l=j}^{+\infty} \frac{l(l-1) \cdots(l-j+1)\left(t^{2}-t|\xi|^{2 \alpha}\right)^{l-j}}{(2 l) !} t^{j} \psi_{j}^{k}(\xi),
\end{aligned}
$$

where

$$
\psi_{j}^{k}(\xi) \preceq|\xi|^{2 j \alpha-k}
$$

Since

$$
\frac{l(l-1) \cdots(l-j+1)}{(2 l) !} \leq \frac{1}{(2(l-j)) !},
$$

we obtain

$$
\begin{aligned}
& \left|\sum_{l=j}^{+\infty} \frac{l(l-1) \cdots(l-j+1)\left(t^{2}-t|\xi|^{2 \alpha}\right)^{l-j}}{(2 l) !}\right| \\
& \quad \leq \sum_{l=j}^{+\infty} \frac{\left|\sqrt{t^{2}-t|\xi|^{2 \alpha}}\right|^{2(l-j)}}{(2(l-j)) !} \preceq \exp \left(\left|\sqrt{t^{2}-t|\xi|^{2 \alpha}}\right|\right) .
\end{aligned}
$$

So (61) is proved. Note by the compact support of $\phi_{2}\left(t^{-1 / 2 \alpha} \xi\right)$, we have

$$
\frac{t}{4} \leq|\xi|^{2 \alpha} \leq 200 t
$$

and we will prove, for all such $\xi$,

$$
\left|e^{-t} \partial_{\xi_{i}}^{k} \cosh \left(\sqrt{t^{2}-t|\xi|^{2 \alpha}}\right)\right| \preceq e^{-b|\xi|^{2 \alpha}} \sum_{j=1}^{k} t^{j}|\xi|^{2 j \alpha-k}, \quad b>0 .
$$

If $t / 4 \leq|\xi|^{2 \alpha} \leq t$, (69) then is a consequence of (61) and (28). If $t<|\xi|^{2 \alpha} \leq 5 t / 4$, then

$$
e^{-t} e^{\sqrt{t|\xi|^{2 \alpha}-t^{2}}}=e^{\sqrt{t|\xi|^{2 \alpha}-t^{2}}-t} \preceq e^{-t / 2} \preceq e^{-2|\xi|^{2 \alpha} / 5} .
$$

When $5 t / 4<|\xi|^{2 \alpha} \leq 200 t$, similar to (33), we get

$$
\begin{aligned}
& e^{-t} \partial_{\xi_{i}}^{k}\left(\cosh \left(i \sqrt{t|\xi|^{2 \alpha}-t^{2}}\right)\right) \\
& \simeq e^{-t} F(t, \xi) \sum_{j=1}^{k} \frac{t^{j} \psi_{j}^{k}(\xi)}{\left(\sqrt{t|\xi|^{2 \alpha}-t^{2}}\right)^{j+\epsilon_{k}}}
\end{aligned}
$$

which is further bounded (note also $t>1$ ) by

$$
e^{-t} \sum_{j=1}^{k} t^{j}|\xi|^{2 j \alpha-k} \preceq e^{-|\xi|^{2 \alpha} / 200} \sum_{j=1}^{k} t^{j}|\xi|^{2 j \alpha-k}
$$

Thus we have proved (69). Fix an $x \in \mathbb{R}^{n}$ and let $x_{i}$ be the variable such that $x_{i}>|x| / n$. Using integration by parts $(n+1)$ times on $\xi_{i}$, we obtain

$$
\begin{aligned}
& t^{n / 2 \alpha} K_{\alpha, m}(t)\left(t^{1 / 2 \alpha} x\right) \\
& \simeq \frac{1}{x_{i}^{n+1}} \sum_{k=0}^{n+1} C_{n+1}^{k} e^{-t} \int_{\mathbb{R}^{n}} \partial_{\xi_{i}}^{n-k+1}\left(\phi_{2}\left(t^{-1 / 2 \alpha} \xi\right)\right) \\
& \quad \times \partial_{\xi_{i}}^{k}\left(\cosh \left(\sqrt{t^{2}-t|\xi|^{2 \alpha}}\right)\right) e^{i\langle\xi, x\rangle} d \xi \\
& \simeq \frac{1}{x_{i}^{n+1}} \sum_{k=1}^{n+1} C_{n+1}^{k} \int_{\mathbb{R}^{n}} \partial_{\xi_{i}^{n-k+1}}\left(\phi_{2}\left(t^{-1 / 2 \alpha} \xi\right)\right) e^{-t} \\
& \quad \times \partial_{\xi_{i}}^{k}\left(\cosh \left(\sqrt{t^{2}-t|\xi|^{2} \alpha}\right)\right) e^{i\langle\xi, x\rangle} d \xi \\
& +\frac{1}{x_{i}^{n+1}} \int_{\mathbb{R}^{n}} \partial_{\xi_{i}}^{n+1}\left(\phi_{2}\left(t^{-1 / 2 \alpha}\right)\right) e^{-t} \\
& \times \cosh \left(\sqrt{t^{2}-t|\xi|^{2 \alpha}}\right) e^{i\langle\xi, x\rangle} d \xi \\
& =K_{1}+K_{2} .
\end{aligned}
$$

By (61), (69), and the compact support of $\phi_{2}$, we have

$\left|K_{1}\right|$

$$
\begin{aligned}
& \leq \frac{1}{|x|^{n+1}} \sum_{k=1}^{n+1} C_{n+1}^{k} \sum_{j=1}^{k} \int_{|\xi| \approx t^{1 / 2 \alpha}}\left|\partial_{\xi_{i}}^{n-k+1} \phi_{2}\left(t^{-1 / 2 \alpha} \xi\right)\right| \\
& \quad \times e^{-b|\xi|^{2 \alpha}} t^{j}|\xi|^{2 j \alpha-k} d \xi \\
& \leq t^{-N / 2 \alpha} \frac{1}{|x|^{n+1}} \sum_{k=1}^{n+1} C_{n+1}^{k} \sum_{j=1}^{k} \int_{|\xi| \approx t^{1 / 2 \alpha}}\left|\phi_{2}^{(n-k+1)}\left(t^{-1 / 2 \alpha} \xi\right)\right| \\
& \preceq t^{-N / 2 \alpha} \frac{1}{|x|^{n+1}} \sum_{k=1}^{n+1} C_{n+1}^{k} \sum_{j=1}^{k} \int_{|\xi| \approx t^{1 / 2 \alpha}}|\xi|^{4 j \alpha+N-n-1} e^{-b|\xi|^{2 \alpha}} d \xi \\
& \preceq t^{-N / 2 \alpha} \frac{1}{|x|^{n+1}} .
\end{aligned}
$$

The second term $K_{2}$ can be calculated directly to finish the whole proof.

By Proposition 9 and the same argument in proving Proposition 8 , we have the following boundedness. 
Proposition 10. Let $f \in H^{r}\left(\mathbb{R}^{n}\right)$. Then for any $t, N>0$ and $r \leq p<+\infty$,

$$
\begin{aligned}
& \left\|K_{\alpha, m}(t) * f\right\|_{H^{p}\left(\mathbb{R}^{n}\right)} \preceq(1+t)^{-N}\|f\|_{H^{r}\left(\mathbb{R}^{n}\right)}, \\
& \left\|\Omega_{\alpha, m}(t) * f\right\|_{H^{p}\left(\mathbb{R}^{n}\right)} \preceq(1+t)^{-N}\|f\|_{H^{r}\left(\mathbb{R}^{n}\right)} .
\end{aligned}
$$

Particularly, we have

$$
\begin{aligned}
& \left\|K_{\alpha, m}(t) * f\right\|_{L^{\infty}\left(\mathbb{R}^{n}\right)} \preceq(1+t)^{-N}\|f\|_{H^{r}\left(\mathbb{R}^{n}\right)}, \\
& \left\|\Omega_{\alpha, m}(t) * f\right\|_{L^{\infty}\left(\mathbb{R}^{n}\right)} \preceq(1+t)^{-N}\|f\|_{H^{r}\left(\mathbb{R}^{n}\right)} .
\end{aligned}
$$

2.3. Estimates for $|\xi|$ near the Infinity. Let $\phi_{3}$ be a $C^{\infty}$ radial function with support in $\left\{\xi \in \mathbb{R}^{n}:|\xi|^{2 \alpha} \geq 2^{6}\right\}$ and satisfy $\phi_{3} \equiv 1$ whenever $|\xi|^{2 \alpha} \geq 100$. Defining

$$
\begin{aligned}
& K_{\alpha, \infty}(t)(x)=e^{-t} \int_{\mathbb{R}^{n}} \phi_{3}(\xi) \cosh \left(i t \sqrt{|\xi|^{2 \alpha}-1}\right) e^{i\langle\xi, x\rangle} d \xi \\
& \Omega_{\alpha, \infty}(t)(x)=e^{-t} \int_{\mathbb{R}^{n}} \phi_{3}(\xi) \frac{\sinh \left(i t \sqrt{|\xi|^{2 \alpha}-1}\right)}{i \sqrt{|\xi|^{2 \alpha}-1}} e^{i\langle\xi, x\rangle} d \xi
\end{aligned}
$$

we have the following proposition.

Proposition 11. Let $1 \leq p \leq+\infty$ and $\alpha>0$. Then there exists a $\delta_{p}>0$ such that for any $\beta>n \alpha|1 / 2-1 / p|$ and $t>0$, we have

$$
\begin{gathered}
\left\|K_{\alpha, \infty}(t) * f\right\|_{L^{p}\left(\mathbb{R}^{n}\right)} \preceq e^{-t}(1+t)^{\delta_{p}}\|f\|_{\dot{L}_{\beta}^{p}}, \\
\left\|\Omega_{\alpha, \infty}(t) * f\right\|_{L^{p}\left(\mathbb{R}^{n}\right)} \preceq e^{-t}(1+t)^{\delta_{p}}\|f\|_{\dot{L}_{\beta-\alpha}^{p}} .
\end{gathered}
$$

Proof. We will show the case $n \geq 2$ and leave the easy case $n=1$ to the reader. Again, we will only show the inequality of $K_{\alpha, \infty}(t) * f$ since the proof of the other one is similar.

Define an analytic family of operators

$$
\begin{aligned}
& T_{z}(f)(x) \\
& \quad=e^{-t} \int_{\mathbb{R}^{n}} \frac{e^{i t \sqrt{|\xi|^{2 \alpha}-1}}}{|\xi|^{z}} \phi_{3}(\xi) \hat{f}(\xi) e^{i\langle x, \xi\rangle} d \xi, \quad z \in \mathbb{C} .
\end{aligned}
$$

By the Plancherel formula, we have

$$
\left\|T_{z}(f)\right\|_{L^{2}\left(\mathbb{R}^{n}\right)} \preceq e^{-t}\|f\|_{L^{2}\left(\mathbb{R}^{n}\right)} \text { for } \operatorname{Re} z=0 \text {. }
$$

If we can show

$$
\left\|T_{z}(f)\right\|_{L^{1}\left(\mathbb{R}^{n}\right)} \preceq(1+t)^{\lambda} e^{-t}\|f\|_{L^{1}\left(\mathbb{R}^{n}\right)}
$$

for $\operatorname{Re} z>n \alpha / 2$ and some $\lambda>0$, the proposition easily follows by a complex interpolation on these two inequalities for $1 \leq p \leq 2$. Then we can use a trivial dual argument to achieve the proposition for the whole range of $p$. Also, without loss of generality, we prove (81) with $z=\beta>n \alpha / 2$.
Let $\Phi$ be a standard cutoff function with support in $\{\xi: 1 / 2 \leq$ $|\xi| \leq 2\}$ satisfying

$$
\sum \Phi\left(2^{-j}|\xi|\right) \equiv 1, \quad \forall \xi \neq 0 .
$$

Defining

$$
\begin{aligned}
& W_{j}^{\alpha}(t) f(x) \\
& \quad=e^{-t} \int_{\mathbb{R}^{n}} e^{i t \sqrt{|\xi|^{2 \alpha}-1}} \Phi\left(2^{-j} \xi\right) \phi_{3}(\xi) \widehat{f}(\xi) e^{i\langle x, \xi\rangle} d \xi,
\end{aligned}
$$

then (81) will follow if we prove

$$
\left\|W_{j}^{\alpha}(t) f(x)\right\|_{L^{1}} \preceq e^{-t}(1+t)^{\lambda} 2^{j n \alpha / 2}\|f\|_{L^{1}} .
$$

In fact, (84) implies

$$
\begin{aligned}
& \sum_{j=0}^{\infty}\left\|2^{-(n \alpha / 2+\epsilon) j} W_{j}^{\alpha}(t) f(x)\right\|_{L^{1}} \\
& \leq \sum_{j=0}^{\infty} 2^{-j \epsilon}(1+t)^{\lambda} e^{-t}\|f\|_{L^{1}}, \quad \forall \epsilon>0 .
\end{aligned}
$$

Noting that $2^{-j} \simeq|\xi|$ in the support of $\Phi\left(2^{-j} \xi\right)$, we get (81) from the above inequality.

Next we prove (84). Let $\Re_{\alpha, j}$ be the kernel of $W_{j}^{\alpha}(t)$. By Young's inequality, it suffices to show

$$
\left\|\mathfrak{R}_{\alpha, j}\right\|_{L^{1}} \preceq e^{-t}(1+t)^{\lambda} 2^{j n \alpha / 2},
$$

for some $\lambda>0$. By the definition, without loss of generality, we may write

$$
\begin{aligned}
\Re_{\alpha, j}(x, t) & =e^{-t} \int_{\mathbb{R}^{n}} \Phi\left(2^{-j} \xi\right) e^{i t \sqrt{|\xi|^{2 \alpha}-1}} e^{i\langle\xi, x\rangle} d \xi \\
& =e^{-t} 2^{j n} \int_{\mathbb{R}^{n}} \Phi(\xi) e^{i t \sqrt{2^{2 \alpha j}|\xi|^{2 \alpha}-1}} e^{i 2^{j}\langle\xi, x\rangle} d \xi .
\end{aligned}
$$

Using the Taylor expansion with integral remainder, for $r \in$ $\operatorname{supp}(\Phi)$, we write

$$
\sqrt{r^{2 \alpha}-1}=r^{\alpha}+g\left(\frac{1}{r}\right)
$$

where

$$
g(r)=-\frac{r^{\alpha}}{2} \int_{0}^{1}\left(1-s r^{2 \alpha}\right)^{-1 / 2} d s
$$

This gives

$$
e^{i t \sqrt{2^{2 j \alpha} r^{2 \alpha}-1}}=e^{i t 2^{j \alpha} r^{\alpha}} e^{i t g\left(1 / r 2^{j \alpha}\right)},
$$

for $2^{\alpha j} \geq 100$ and $1 / 2 \leq r \leq 2$. By the definition of $g$ it is easy to see that for any integer $m \geq 0$

$$
\left|\frac{d^{m}}{d r^{m}} e^{i t g\left(1 / 2^{k \alpha} r\right)}\right| \preceq(1+t)^{m}
$$

uniformly for $2^{\alpha j} \geq 100$ and $1 / 2 \leq r \leq 2$. 
Now we write

$$
\Re_{\alpha, j}(x, t)=e^{-t} 2^{j n} \int_{\mathbb{R}^{n}} e^{i \wp(\xi, t, j)} \Phi(\xi) e^{i t g\left(1 / 2^{j \alpha}|\xi|\right)} d \xi,
$$

where the phase function $\wp$ is defined as

$$
\wp(\xi, t, j)=t 2^{j \alpha}|\xi|^{\alpha}+2^{j}\langle\xi, x\rangle .
$$

Let sets $E_{1}, E_{2}$, and $E_{3}$ be defined as

$$
\begin{aligned}
& E_{1}=\left\{x \in \mathbb{R}^{n}:|x| \geq M t 2^{j(\alpha-1)}\right\}, \\
& E_{2}=\left\{x \in \mathbb{R}^{n}:|x| \leq m t 2^{j(\alpha-1)}\right\}, \\
& E_{3}=\left\{x \in \mathbb{R}^{n}: m t 2^{j(\alpha-1)}<|x|<M t 2^{j(\alpha-1)}\right\},
\end{aligned}
$$

where

$$
M=n 2^{\alpha+100} \max \left\{\alpha, \frac{1}{\alpha}\right\}
$$

and $m=1 / M$. Hence,

$$
\left\|\mathfrak{R}_{\alpha, j}\right\|_{L^{1}}=\left\|\chi_{E_{1}} \mathfrak{R}_{\alpha, j}\right\|_{L^{1}}+\left\|\chi_{E_{2}} \mathfrak{R}_{\alpha, j}\right\|_{L^{1}}+\left\|\chi_{E_{3}} \mathfrak{R}_{\alpha, j}\right\|_{L^{1}},
$$

where $\chi_{E}$ denotes the characteristic function of a set $E$.

Furthermore, we let

$$
E_{1, m}=\left\{x \in E_{1}:\left|x_{m}\right| \geq\left|x_{i}\right| \text { for } i=1,2, \ldots, n\right\},
$$

for $m=1,2, \ldots, n$. Then

$$
\left\|\chi_{E_{1}} \mathfrak{R}_{\alpha, j}\right\|_{L^{1}} \leq \sum_{m=1}^{n}\left\|\chi_{E_{1, m}} \mathfrak{R}_{\alpha, j}\right\|_{L^{1}} .
$$

For each $\chi_{E_{1, m}} \mathfrak{R}_{\alpha, j}$, using integration by parts on the $\xi_{m}$ variable, it is easy to obtain that, for $m=1,2, \ldots, n$,

$$
\left|\chi_{E_{1, m}}(x) \Re_{\alpha, j}(x, t)\right| \preceq e^{-t} 2^{j n} \chi_{E_{1, m}}(x) \min \left\{1,\left(2^{j}|x|\right)^{-N}\right\},
$$

for any positive number $N$.

By the polar decomposition,

$$
\begin{aligned}
\chi_{E_{2}} & (x) \mathfrak{R}_{\alpha, j}(x, t) \\
& =e^{-t} 2^{j n} \int_{S^{n-1}}\left(\int_{0}^{\infty} \Phi_{0}(r) e^{i P(r, t, j)} d r\right) d \sigma\left(\xi^{\prime}\right),
\end{aligned}
$$

where the phase function $P$ is defined by

$$
\begin{gathered}
P(r, t, j)=t 2^{j \alpha} r^{\alpha}+2^{j} r\left\langle\xi^{\prime}, x\right\rangle, \\
\Phi_{0}(r)=\Phi(r) e^{i t g\left(1 / 2^{j \alpha} r\right)} .
\end{gathered}
$$

Using integration by parts on the inner integral, we obtain

$$
\left|\chi_{E_{2}}(x) \Re_{\alpha, j}(x, t)\right| \preceq e^{-t} 2^{j n} \chi_{E_{2}}(x) \min \left\{1,\left(t 2^{j \alpha}\right)^{-N}\right\},
$$

for any positive number $N$.
By the Proposition in [48, page 344],

$$
\left|\chi_{E_{3}}(x) \Re_{\alpha, j}(x, t)\right| \preceq e^{-t} 2^{j n} \chi_{E_{3}}(x) \min \left\{1,\left(t 2^{j \alpha}\right)^{-n / 2}\right\} .
$$

Thus, if $t 2^{j \alpha} \geq 1$

$$
\begin{aligned}
\left\|\chi_{E_{3}} \mathfrak{R}_{\alpha, j}\right\|_{L^{1}} & \preceq e^{-t} 2^{j n} \int_{|x| \simeq t 2^{j(\alpha-1)}}\left(t 2^{j \alpha}\right)^{-n / 2} d x \\
& \preceq e^{-t} t^{n / 2} 2^{j \alpha n / 2} .
\end{aligned}
$$

If $t 2^{j \alpha} \leq 1$

$$
\begin{aligned}
\left\|\chi_{E_{3}} \mathfrak{R}_{\alpha, j}\right\|_{L^{1}} & \preceq e^{-t} 2^{j n} \int_{|x| \simeq t 2^{j(\alpha-1)}} d x \\
& \preceq e^{-t}\left(t 2^{j \alpha}\right)^{n} \leq e^{-t} .
\end{aligned}
$$

For $\chi_{E_{2}} \mathfrak{R}_{\alpha, j}$, if $t 2^{j \alpha} \leq 1$,

$$
\begin{aligned}
\left\|\chi_{E_{2}} \mathfrak{R}_{\alpha, j}\right\|_{L^{1}} & \preceq e^{-t} 2^{j n} \int_{|x| \leq t 2^{j(\alpha-1)}} d x \\
& \preceq e^{-t}\left(t 2^{j \alpha}\right)^{n} \preceq e^{-t} .
\end{aligned}
$$

If $t 2^{j \alpha}>1$ and then we choose $N=n$,

$$
\begin{aligned}
\left\|\chi_{E_{2}} \mathfrak{R}_{\alpha, j}\right\|_{L^{1}} & \preceq e^{-t} 2^{j n}\left(t 2^{j \alpha}\right)^{-N}\left(t 2^{j(\alpha-1)}\right)^{n} \\
& =e^{-t}\left(t 2^{j \alpha}\right)^{-N} t^{n} 2^{j n \alpha} \preceq e^{-t} .
\end{aligned}
$$

Finally we estimate $\chi_{E_{1}} \mathfrak{R}_{\alpha, j}$. For each $m=1,2, \ldots, n$,

$$
\left\|\chi_{E_{1, m}} \mathfrak{R}_{\alpha, j}\right\|_{L^{1}} \preceq e^{-t} 2^{j n} \int_{|x| \geq t 2^{j(\alpha-1)}} \min \left\{1,\left(2^{j}|x|\right)^{-N}\right\} d x .
$$

If the set $\left\{x: t 2^{j(\alpha-1)} \preceq|x| \preceq 2^{-j}\right\}$ is not empty, we write

$$
\begin{aligned}
\left\|\chi_{E_{1, m}} \mathfrak{R}_{\alpha, j}\right\|_{L^{1}} \preceq & e^{-t} 2^{j n} \int_{t 2^{j(\alpha-1)} \leq|x| \leq 2^{-j}} d x \\
& +e^{-t} 2^{j n} \int_{2^{-j} \leq|x|} \min \left\{1,\left(t 2^{j \alpha}\right)^{-N}\right\} d x \\
& =J_{1}+J_{2} .
\end{aligned}
$$

Clearly

$$
J_{1} \leq e^{-t}
$$

Also, choose a sufficiently large $N$, and then

$$
J_{2} \preceq e^{-t} 2^{j n} \int_{|x| \geq 2^{-j}}\left(2^{j}|x|\right)^{-N} d x \preceq e^{-t} .
$$

If the set $\left\{2^{-j} \geq|x| \geq t 2^{j(\alpha-1)}\right\}$ is empty, then we also have

$$
\left\|\chi_{E_{1, m}} \mathfrak{R}_{\alpha, j}\right\|_{L^{1}} \preceq e^{-t} 2^{j n} \int_{|x| \geq 2^{-j}}\left(2^{j}|x|\right)^{-N} d x \preceq e^{-t} .
$$

The proposition is proved. 


\section{Proof of Theorems 2 and 3}

Proof of Theorem 2. Recalling the definition of

$$
K_{\alpha, 0}(t), K_{\alpha, m}(t), K_{\alpha, \infty}(t), \quad \Omega_{\alpha, 0}(t), \Omega_{\alpha, m}(t), \Omega_{\alpha, \infty}(t)
$$

in Section 2, we have

$$
\begin{aligned}
& \left|K_{\alpha}(t) * f\right| \\
& \quad \leq\left|K_{\alpha, 0}(t) * f\right|+\left|K_{\alpha, m}(t) * f\right|+\left|K_{\alpha, \infty}(t) * f\right|, \\
& \left|\Omega_{\alpha}(t) * f\right| \\
& \quad \leq\left|\Omega_{\alpha, 0}(t) * f\right|+\left|K_{\alpha, m}(t) * f\right|+\left|\Omega_{\alpha, \infty}(t) * f\right| .
\end{aligned}
$$

By the triangle inequality and Propositions 8,10 , and 11 , we only have to verify that, for any $n / 2 \alpha$-admissible triplet $(p, q, r)$,

$$
\begin{aligned}
& \left(\int_{0}^{\infty}\left\|K_{\alpha, 0}(t) * f\right\|_{L^{p}\left(\mathbb{R}^{n}\right)}^{q} d t\right)^{1 / q} \leq\|f\|_{H^{r}\left(\mathbb{R}^{n}\right)}, \\
& \left(\int_{0}^{\infty}\left\|\Omega_{\alpha, 0}(t) * f\right\|_{L^{p}\left(\mathbb{R}^{n}\right)}^{q} d t\right)^{1 / q} \leq\|f\|_{H^{r}\left(\mathbb{R}^{n}\right)} .
\end{aligned}
$$

These two inequalities are obviously true if

$$
\frac{1}{q}<\frac{n}{2 \alpha}\left(\frac{1}{r}-\frac{1}{p}\right)
$$

For $1 / q=(n / 2 \alpha)(1 / r-1 / p)$, denote

$$
F(t) f=\left\|K_{\alpha, 0}(t) * f\right\|_{L^{p}\left(\mathbb{R}^{n}\right)} .
$$

By Proposition 8, we have

$$
F(t) f \preceq(1+t)^{-1 / q}\|f\|_{H^{r}} .
$$

This indicates that, for any $\lambda>0$, there exists a positive constant $C$ independent of $\lambda$ and $f$ such that

$$
\begin{aligned}
& |\{t:|F(t) f|>\lambda\}| \\
& \quad \leq\left|\left\{t: C t^{-1 / q}\|f\|_{H^{r}}>\lambda\right\}\right| \leq\left(\frac{C\|f\|_{H^{r}}}{\lambda}\right)^{q} .
\end{aligned}
$$

This shows that $K_{\alpha, 0}(t)$ is a bounded mapping from $H^{r}\left(\mathbb{R}^{n}\right)$ to the mixed norm space $L^{q, \infty}\left([0, \infty], L^{p}\left(\mathbb{R}^{n}\right)\right)$ for any admissible triplet $(p, q, r)$. Now we choose admissible triplets $\left(p, q_{1}, r_{1}\right)$ and $\left(p, q_{2}, r_{2}\right)$ satisfying

$$
\begin{array}{ll}
r_{1}<r<r_{2}<\infty, & q_{1}<q<q_{2}<\infty, \\
\frac{1}{q}=\frac{\theta}{q_{1}}+\frac{1-\theta}{q_{2}}, & \frac{1}{r}=\frac{\theta}{r_{1}}+\frac{1-\theta}{r_{2}} .
\end{array}
$$

Then by the Marcinkiewicz interpolation, we easily obtain

$$
\left(\int_{0}^{\infty}\left\|K_{\alpha, 0}(t) * f\right\|_{L^{p}\left(\mathbb{R}^{n}\right)}^{q} d t\right)^{1 / q} \leq\|f\|_{H^{r}\left(\mathbb{R}^{n}\right)} .
$$

Similarly we can show that, for any $n / 2 \alpha$-admissible triplet $(p, q, r)$,

$$
\left(\int_{0}^{\infty}\left\|\Omega_{\alpha, 0}(t) * f\right\|_{L^{p}\left(\mathbb{R}^{n}\right)}^{q} d t\right)^{1 / q} \leq\|f\|_{H^{r}\left(\mathbb{R}^{n}\right)} .
$$

Proof of Theorem 3. By checking the above proof, we only need to show the following proposition.

Proposition 12. There is a $\delta_{p}>0$ for which if $\beta>(n-1) \mid 1 / p-$ $1 / 2 \mid$, then

$$
\begin{aligned}
& \left\|K_{1, \infty} * f\right\|_{L^{p}\left(\mathbb{R}^{n}\right)} \preceq(1+t)^{\delta_{p}} e^{-t}\|f\|_{\dot{L}_{\beta}^{p}\left(\mathbb{R}^{n}\right)}, \\
& \left\|\Omega_{1, \infty} * f\right\|_{L^{p}\left(\mathbb{R}^{n}\right)} \preceq(1+t)^{\delta_{p}} e^{-t}\|f\|_{\dot{L}_{\beta-1}^{p}\left(\mathbb{R}^{n}\right)}
\end{aligned}
$$

hold for all $1 \leq p \leq \infty$.

Proof. Let

$$
W_{\beta}(t) f(x)=e^{-t} \int_{\mathbb{R}^{n}} \frac{e^{i t \sqrt{|\xi|^{2}-1}} \phi_{3}(\xi)}{|\xi|^{\beta}} \widehat{f}(\xi) e^{i\langle x, \xi\rangle} d \xi,
$$

where $\phi_{3}$ is defined in Section 2.3 (corresponding to $\alpha=1$ ). We will prove, for any $\beta>(n-1) / 2$, that

$$
\left\|W_{\beta}(t) f(x)\right\|_{L^{1}} \preceq e^{-t}(1+t)^{\lambda}\|f\|_{L^{1}},
$$

with some $\lambda>0$. Then by repeating the complex interpolation argument in the proof of Proposition 11, with (81) replaced by (125), we finish the proof of the proposition.

Next we turn to the proof of (125). Denote the kernel of $W_{\beta}(t)$ by

$$
\Im_{\beta}(x, t)=e^{-t} \int_{\mathbb{R}^{n}} \frac{e^{i t \sqrt{|\xi|^{2}-1}} \phi_{3}(\xi)}{|\xi|^{\beta}} e^{i\langle x, \xi\rangle} d \xi .
$$

By Young's inequality, it suffices to show that if $\beta>(n-1) / 2$, then

$$
\left\|\varsigma_{\beta}(x, t)\right\|_{L^{1}} \preceq(1+t)^{(n+1) / 2} e^{-t} .
$$

Let $\Phi$ be the cutoff function defined in Section 2.3. Then we have

$$
\begin{aligned}
\Im_{\beta}(x, t) & =e^{-t} \sum_{k=6}^{\infty} \int_{\mathbb{R}^{n}} \frac{e^{i t \sqrt{|\xi|^{2}-1}}}{|\xi|^{\beta}} \Phi\left(2^{-k}|\xi|\right) \phi_{3}(|\xi|) e^{i\langle\xi, x\rangle} d \xi \\
& =e^{-t} \sum_{k=6}^{\infty} Y_{k}(x),
\end{aligned}
$$

where, by [49, Ch. 4],

$$
\begin{aligned}
Y_{k}(x) & =\int_{\mathbb{R}^{n}} \frac{e^{i t \sqrt{|\xi|^{2}-1}}}{|\xi|^{\beta}} \Phi\left(2^{-k}|\xi|\right) \phi_{3}(|\xi|) e^{i\langle\xi, x\rangle} d \xi \\
& \simeq \int_{0}^{\infty} \frac{e^{i t \sqrt{r^{2}-1}}}{r^{\beta}} \phi_{3}(r) \Phi\left(2^{-k} r\right) V_{(n-2) / 2}(r|x|) r^{n-1} d r .
\end{aligned}
$$


In the last integral,

$$
V_{v}(s)=\frac{J_{\nu}(s)}{s^{v}}
$$

and $J_{\nu}(s)$ is the Bessel function of order $\nu$.

So, by the Minkowski inequality,

$$
\left\|\varsigma_{\beta}(\cdot, t)\right\|_{L^{1}} \leq e^{-t} \sum_{k=6}^{\infty}\left\|Y_{k}\right\|_{L^{1}} \text {. }
$$

First, we assume $t \geq 1$. Changing variables, we have

$$
\begin{aligned}
& Y_{k}(x) \\
& =\frac{|x|^{(2-n) / 2}}{2^{k(\beta-n / 2-1)}} \int_{0}^{\infty} e^{i t \sqrt{2^{2 k} r^{2}-1}} \Phi(r) \phi_{3}\left(2^{k} r\right) J_{(n-2) / 2} \\
& \quad \times\left(2^{k} r|x|\right) r^{n / 2-\beta-1 / 2} d r .
\end{aligned}
$$

Using the Taylor expansion with integral remainder, for $r \in \operatorname{supp}\left(\phi_{3}\right)$, we write

$$
\sqrt{r^{2}-1}=r+g(r)
$$

where

$$
g\left(\frac{1}{r}\right)=-\frac{1}{2 r} \int_{0}^{1}\left(1-\frac{s}{r^{2}}\right)^{-1 / 2} d s
$$

This gives

$$
e^{i t \sqrt{2^{2 k} r^{2}-1}}=e^{i t 2^{k} r} e^{i t g\left(1 / 2^{k} r\right)},
$$

for $k \geq 6$ and $1 / 2 \leq r \leq 2$. By the definition of $g$ it is easy to see that if we denote $h(r)=g\left(1 / 2^{k} r\right)$, then

$$
\left|h^{(m)}(r)\right| \preceq 2^{-k} .
$$

Also, for any integer $m \geq 0$,

$$
\begin{aligned}
\left|\frac{d^{m}}{d r^{m}} e^{i t g\left(2^{k} r\right)}\right| & \preceq 1 \quad \text { if } t 2^{-k} \leq 1, \\
\left|\frac{d^{m}}{d r^{m}} e^{i t g\left(2^{k} r\right)}\right| & \preceq\left(t 2^{-k}\right)^{m} \quad \text { if } t 2^{-k}>1
\end{aligned}
$$

uniformly for $k \geq 10$ and $1 / 2 \leq r \leq 2$.

When

$$
2^{k}|x| \leq 2^{4}
$$

using the known estimate

$$
J_{(n-2) / 2}(r)=O\left(r^{(n-2) / 2}\right), \quad \text { as } r \longrightarrow 0,
$$

it is easy to see

$$
\left|Y_{k}(x)\right| \preceq|x|^{(2-n) / 2} 2^{-k(\beta-n / 2-1)}\left(2^{k}|x|\right)^{(n-2) / 2}=2^{-k(\beta-n)} .
$$

Thus,

$$
e^{-t} \sum_{k=6}^{\infty}\left\|Y_{k} \chi_{\left\{|x| \leq 2^{-k+4}\right\}}\right\|_{L^{1}} \preceq e^{-t} \sum_{k=6}^{\infty} 2^{-k(\beta-n)} \int_{|x| \leq 2^{-k+4}} d x \preceq e^{-t}
$$

When

$$
2^{k}|x|>2^{4}
$$

we use the asymptotic expansion of $J_{(n-2) / 2}(r)$ : for any integer $N \geq 0$,

$$
J_{\nu}(r) \simeq e^{ \pm i r}\left(\sum_{j=0}^{N} \frac{c_{j}}{r^{j+1 / 2}}\right)+O\left(r^{-(N+1)-1 / 2}\right),
$$

where $c_{1}, c_{2}, \ldots, c_{N}$ are constants.

$$
\text { In this case, }
$$

$$
\begin{aligned}
Y_{k}(x) & \\
\simeq & \sum_{j=0}^{N} c_{j} \frac{|x|^{(1-n) / 2-j}}{2^{k(\beta-n / 2-1 / 2+j)}} \\
& \times \int_{0}^{\infty} e^{i 2^{k} r(t \pm|x|)} e^{i t g\left(2^{k} r\right)} \Phi(r) \phi_{3}\left(2^{k} r\right) r^{n / 2-\beta-j} d r \\
& +O\left(|x|^{(-n+1) / 2-N-1} 2^{-k(\beta-n / 2+1 / 2+N)}\right) \\
= & \sum_{j=0}^{N} c_{j} Y_{k, j}(x)+O\left(|x|^{(-n+1) / 2-N-1} 2^{-k(\beta-n / 2+1 / 2+N)}\right),
\end{aligned}
$$

where, without loss of generality, we denote

$$
\begin{aligned}
Y_{k, j}(x) & \\
= & \frac{|x|^{(1-n) / 2-j}}{2^{k(\beta-n / 2-1 / 2+j)}} \\
& \quad \times \int_{0}^{\infty} e^{i 2^{k} r(t-|x|)} e^{i t g\left(2^{k} r\right)} \Phi(r) \phi_{3}\left(2^{k} r\right) r^{n / 2-\beta-j} d r .
\end{aligned}
$$

It is easy to see that, for a suitable integer $N$,

$$
e^{-t} \sum_{k=6}^{\infty} 2^{-k(\beta-n / 2+1 / 2+N)} \int_{|x| \geq 2^{-k+4}}|x|^{(-n+1) / 2-N-1} d x \preceq e^{-t} .
$$

Thus it remains to show that, for each $j$,

$$
e^{-t} \sum_{k=6}^{\infty}\left\|Y_{k, j} \chi_{\left\{|x|>2^{-k+4}\right\}}\right\|_{L^{1}} \preceq t^{(n+1) / 2} e^{-t} .
$$

Since the estimates of all $Y_{k, j}$ are similar, we will only show

$$
e^{-t} \sum_{k=6}^{\infty}\left\|Y_{k, 0} \chi_{\left\{|x|>2^{-k+4}\right\}}\right\|_{L^{1}} \preceq t^{(n+1) / 2} e^{-t}
$$


Using integration by parts and noting $\phi_{3}\left(2^{k} r\right) \equiv 1$ if $k>2^{8}$ and $r \in \operatorname{supp}(\Phi)$, it is easy to check that one has

$$
\begin{aligned}
& \left|\int_{0}^{\infty} e^{i 2^{k} r(t-|x|)} e^{i t g\left(2^{k} r\right)} \Phi(r) \Psi\left(2^{k} r\right) r^{n / 2-\beta-j} d r\right| \\
& \quad \leq \min \left\{1,\left.2^{-m k}|t-| x\right|^{-m}\left(2^{-k} t\right)^{m}\right\}
\end{aligned}
$$

if $2^{-k} t>1$, for any positive integer $m$, and

$$
\begin{aligned}
& \left|\int_{0}^{\infty} e^{i 2^{k} r(t \pm|x|)} e^{i t g\left(2^{k} r\right)} \Phi(r) \phi_{3}\left(2^{k} r\right) r^{n / 2-\beta-j} d t\right| \\
& \quad \leq \min \left\{1,\left.2^{-\mu k}|t-| x\right|^{-\mu}\right\}
\end{aligned}
$$

if $2^{-k} t \leq 1$, for any positive integer $\mu$. Thus, we have the following lemma.

Lemma 13. Let $2^{k}|x| \geq 10$. For any $m \geq 0$, one has

$$
\left|Y_{k, 0}(x)\right| \leq|x|^{(1-n) / 2} 2^{-k(\beta-n / 2-1 / 2)} 2^{-m k}|t-| x||^{-m}
$$

if $2^{-k} t \leq 1$.

Also, for any $\mu \geq 0$,

$$
\left|Y_{k, 0}(x)\right| \leq|x|^{(1-n) / 2} 2^{-k(\beta-n / 2-1 / 2)} 2^{-\mu k}|t-| x||^{-\mu}\left(2^{-k} t\right)^{\mu}
$$

if $2^{-k} t>1$.

Now we continue the proof of the proposition. Write

$$
\begin{aligned}
& \sum_{k=6}^{\infty}\left\|Y_{k, 0} \chi_{\left\{|x|>2^{-k+4}\right\}}\right\|_{L^{1}\left(\mathbb{R}^{n}\right)} \\
& \leq \sum_{k=6}^{\infty} \int_{10 / 2^{k}<|x| \leq t / 2}\left|Y_{k, 0}(x)\right| d x \\
& \quad+\sum_{k=6}^{\infty} \int_{|x|>100 t}\left|Y_{k, 0}(x)\right| d x \\
& \quad+\sum_{k=6}^{\infty} \int_{t / 2<|x| \leq 100 t}\left|Y_{k, 0}(x)\right| d x \\
& =A_{1}+A_{2}+A_{3} .
\end{aligned}
$$

In $A_{1}$, noting $\beta-(n-1) / 2>0$, we use the lemma with $\mu=1 / 2$ and $m=1$ :

$$
\begin{aligned}
A_{1} & \leq \sum_{k=6}^{\log t} 2^{-k(\beta-n / 2+1 / 2)} \int_{10 / 2^{k}<|x| \leq t / 2}|x|^{(-n+1) / 2} d x \\
& +t^{-1} \sum_{k=\log t+1}^{\infty} 2^{-k(\beta-n / 2+1 / 2)} \int_{10 / 2^{k}<|x| \leq t / 2}|x|^{(-n+1) / 2} d x \\
& \leq t^{(n+1) / 2} .
\end{aligned}
$$

Similarly, in Lemma 13 we let $\mu=m=n$ :

$$
\begin{aligned}
A_{2} \preceq & \sum_{k=6}^{\log t} 2^{-k(\beta-n / 2-1 / 2)} t^{n} \int_{100 t<|x|}|x|^{(1-n) / 2-n} 2^{-2 n k} d x \\
& +\sum_{k=\log t+1}^{\infty} 2^{-k(\beta-n / 2-1 / 2)} \int_{100 t<|x|} 2^{-n k}|x|^{(1-n) / 2-n} d x \\
& \leq t^{(n+1) / 2} .
\end{aligned}
$$

Let

$$
\begin{aligned}
E & =\left\{x \in \mathbb{R}^{n}: \frac{t}{2}<|x| \leq 100 t\right\}, \\
E_{k} & =\left\{x \in \mathbb{R}^{n}:|t-| x||<2^{-k}\right\} .
\end{aligned}
$$

Using Lemma 13, we write

$$
\begin{aligned}
A_{3} \preceq & \sum_{k=6}^{\log t} 2^{-k(\beta-n / 2-1 / 2)} \\
& \times \int_{E \backslash E_{k}}|x|^{(1-n) / 2} 2^{-\mu k}|t-| x||^{-\mu}\left(2^{-k} t\right)^{\mu} d x \\
& +\sum_{k=\log t+1}^{\infty} 2^{-k(\beta-n / 2-1 / 2)} \\
& \times \int_{E \backslash E_{k}}|x|^{(1-n) / 2} 2^{-m k}|t-| x||^{-m} d x \\
& +\sum_{k=6}^{\infty} 2^{-k(\beta-n / 2-1 / 2)} \int_{E_{k}}|x|^{(1-n) / 2} d x \\
= & B_{1}+B_{2}+B_{3} .
\end{aligned}
$$

Here, the last term

$$
\begin{aligned}
B_{3} & =\sum_{k=6}^{\infty} 2^{-k(\beta-n / 2-1 / 2)} \int_{E_{k}}|x| \frac{1-n}{2} d x \\
& \preceq \sum_{k=6}^{\infty} 2^{-k(\beta-n / 2-1 / 2)} \int_{t-2^{-k}}^{t+2^{-k}} r^{(1-n) / 2+n-1} \preceq t^{(n+1) / 2} .
\end{aligned}
$$

Use the polar coordinate and Lemma 13 for $\mu=1 / 2$ :

$$
\begin{array}{r}
B_{1}=\sum_{k=6}^{\log t} 2^{-k(\beta-n / 2+1 / 2)} t^{1 / 2} \int_{E \backslash E_{k}}|x|^{(1-n) / 2}|t-| x||^{-1 / 2} d x \\
\preceq \sum_{k=6}^{\log t} 2^{-k(\beta-n / 2+1 / 2)} t^{1 / 2}\left(\int_{t+2^{-k}}^{100 t} r^{(n-1) / 2}(r-t)^{-1 / 2} d r\right. \\
\left.+\int_{t / 2}^{t-2^{-k}} r^{(n-1) / 2}(r-t)^{-1 / 2} d r\right)
\end{array}
$$


Abstract and Applied Analysis

13

$$
\begin{aligned}
& \leq t^{n / 2} \sum_{k=6}^{\log t} 2^{-k(\beta-n / 2+1 / 2)}\left(\int_{t+2^{-k}}^{100 t}(r-t)^{-1 / 2} d r\right. \\
& \left.\quad+\int_{t / 2}^{t-2^{-k}}(r-t)^{-1 / 2} d r\right) \\
& \leq t^{(n+1) / 2} .
\end{aligned}
$$

Similarly, we can show

$$
\begin{aligned}
& \sum_{k=\log t+1}^{\infty} 2^{-k(\beta-n / 2-1 / 2)} \int_{E \backslash E_{k}}|x|^{(1-n) / 2} 2^{-m k}|t-| x||^{-m} d x \\
& \leq t^{(n+1) / 2} .
\end{aligned}
$$

When $0<t \leq 1$, the proof is the same with only minor modifications.

4. Almost Everywhere Convergence

Next we will study the pointwise convergence of the solution $u(t, x)$ of $(5)$ to the initial data. We will prove the following.

Theorem 14. Let $s>1 / 2$. If $f$ belongs to the inhomogeneous Sobolev space $L_{s}^{2}\left(\mathbb{R}^{n}\right)$ and $g \in L_{s-\alpha}^{2}\left(\mathbb{R}^{n}\right)$, then the solution $u(t, x)$ of (5) converges to $f(x)$ are. $x \in \mathbb{R}^{n}$ as $t \rightarrow 0^{+}$.

To prove this theorem, we need Lemma 15 and Proposition 16.

Lemma 15 (see [50]). Let $n \geq 2$ and $1<d<n$. Then

$$
\int_{\mathbb{R}^{n}}\left|\int_{S^{n-1}} g(u) e^{i x \cdot u} d \sigma(u)\right|^{2} \frac{d x}{|x|^{d}} \preceq \int_{S^{n-1}}|g(u)|^{2} d \sigma(u) .
$$

Proposition 16. Let $n \geq 2$ and let $m(t,|\xi|)$ be defined on $\mathbb{R}^{+} \times$ $\mathbb{R}^{n}$ and satisfy

$$
\left|(1+|\xi|)^{\gamma} m(t,|\xi|)\right| \preceq 1
$$

Denote the maximal function

$$
m^{*} f(x)=\sup _{t>0}|m(t, D) f(x)| .
$$

Then if $\gamma>0$, we have

$$
\left\|m^{*} f(x)\right\|_{L^{2}\left(|x|^{-d} d x\right)} \preceq\|f\|_{\dot{L}_{l}^{2}}, \quad \frac{d-2 \gamma}{2}<l<\frac{d}{2}, d>1 .
$$

If $\gamma \leq 0$, then

$$
\left\|m^{*} f(x)\right\|_{L^{2}\left(|x|^{-d} d x\right)} \preceq\|f\|_{L_{l}^{2}}, \quad l>d-\gamma-\frac{1}{2}, d>1 .
$$

Proof. Making $t$ into a function of $x$, we only have to bound

$$
m(t(x), D) f(x)=\int_{\mathbb{R}^{n}} e^{i\langle x, \xi\rangle} m(t(x),|\xi|) \widehat{f}(\xi) d \xi
$$

where $t(x): \mathbb{R}^{n} \rightarrow \mathbb{R}^{+}$is any measurable function. By the polar decomposition,

$$
\begin{aligned}
& |m(t(x), D) f(x)| \\
& \quad=\left|\int_{0}^{\infty} r^{n-1} m(t(x), r) \int_{S^{n-1}} \widehat{f}\left(r \xi^{\prime}\right) e^{i\left\langle r x, \xi^{\prime}\right\rangle} d \sigma\left(\xi^{\prime}\right) d r\right| \\
& \quad \preceq \int_{0}^{\infty} r^{n-1}(1+r)^{-\gamma}\left|\int_{S^{n-1}} \widehat{f}\left(r \xi^{\prime}\right) e^{i\left\langle r x, \xi^{\prime}\right\rangle} d \sigma\left(\xi^{\prime}\right)\right| d r .
\end{aligned}
$$

By Minkowski's inequality, change of variables, and Lemma 15, we have

$$
\begin{aligned}
& \|m(t(x), D) f(x)\|_{L^{p}\left(|x|^{-d} d x\right)} \\
& \preceq \int_{0}^{\infty} r^{n-1}(1+r)^{-\gamma} \\
& \quad \times\left\|\int_{S^{n-1}} \widehat{f}\left(r \xi^{\prime}\right) e^{i\left\langle r x, \xi^{\prime}\right\rangle} d \sigma\left(\xi^{\prime}\right)\right\|_{L^{2}\left(d x /|x|^{d}\right)} d r \\
& =\int_{0}^{\infty} r^{n / 2+d / 2-1}(1+r)^{-\gamma} \\
& \quad \times\left\|\int_{S^{n-1}} \hat{f}\left(r \xi^{\prime}\right) e^{i\left\langle x, \xi^{\prime}\right\rangle} d \sigma\left(\xi^{\prime}\right)\right\|_{L^{2}\left(d x /|x|^{d}\right)} d r \\
& =\left(\int_{0}^{\infty}+\int_{2}^{\infty / 2+d / 2-1}(1+r)^{-\gamma}\left(\int_{S^{n-1}}\left|\widehat{f}\left(r \xi^{\prime}\right)\right|^{2} d \sigma\left(\xi^{\prime}\right)\right)^{1 / 2} d r\right. \\
& \quad \times\left(\int_{S^{n-1}}\left|\widehat{f}\left(r \xi^{\prime}\right)\right|^{2} d \sigma\left(\xi^{\prime}\right)\right)^{1 / 2} d r \\
& =L_{1}+L_{2} \cdot
\end{aligned}
$$

When $\gamma>0$, we have

$$
\begin{aligned}
L_{1} & \leq \int_{0}^{2} r^{n / 2+d / 2-1}\left(\int_{S^{n-1}}\left|\widehat{f}\left(r \xi^{\prime}\right)\right|^{2} d \sigma\left(\xi^{\prime}\right)\right)^{1 / 2} d r \\
& \left(\int_{0}^{2} r^{d-1-2 l} d r\right)^{1 / 2} \\
& \times\left(\int_{0}^{2} r^{2 l} r^{n-1} \int_{S^{n-1}}\left|\widehat{f}\left(r \xi^{\prime}\right)\right|^{2} d \sigma\left(\xi^{\prime}\right) d r\right)^{1 / 2} \\
& \leq\left(\int_{0}^{2} r^{d-1-2 l} d r\right)^{1 / 2}\left(\int_{|\xi| \leq 2}|\xi|^{2 l}|\widehat{f}(\xi)|^{2} d \xi\right)^{1 / 2} \\
& \leq\|f\|_{\dot{L}_{l}^{2}\left(\mathbb{R}^{n}\right)^{\cdot}}
\end{aligned}
$$


Here we have to let

$$
d-1-2 l<-1
$$

On the other hand,

$$
\begin{aligned}
L_{2} & \leq \int_{2}^{\infty} r^{n / 2+d / 2-1-\gamma}\left(\int_{S^{n-1}}\left|\widehat{f}\left(r \xi^{\prime}\right)\right|^{2} d \sigma\left(\xi^{\prime}\right)\right)^{1 / 2} d r \\
& \leq\left(\int_{2}^{\infty} r^{d-1-2 l-2 \gamma} d r\right)^{1 / 2} \\
& \times\left(\int_{2}^{\infty} r^{2 l} r^{n-1} \int_{S^{n-1}}\left|\widehat{f}\left(r \xi^{\prime}\right)\right|^{2} d \sigma\left(\xi^{\prime}\right) d r\right)^{1 / 2} \\
& \leq\left(\int_{2}^{\infty} r^{d-1-2 l-2 \gamma} d r\right)^{1 / 2}\left(\int_{|\xi| \geq 2}|\xi|^{2 l}|\widehat{f}(\xi)|^{2} d \xi\right)^{1 / 2} \\
\leq & \|f\|_{\dot{L}_{l}^{2}\left(\mathbb{R}^{n}\right)}
\end{aligned}
$$

Obviously we have to let

$$
d-1-2 l-2 \gamma>-1,
$$

which, together with (170), implies

$$
\frac{d-2 \alpha}{2}<l<\frac{d}{2} \text {. }
$$

If $\gamma \leq 0$, then

$$
\begin{aligned}
L_{1} \leq & \left(\int_{0}^{2} r^{d-1} d r\right)^{1 / 2} \\
& \times\left(\int_{0}^{2} r^{n-1} \int_{S^{n-1}}\left|\widehat{f}\left(r \xi^{\prime}\right)\right|^{2} d \sigma\left(\xi^{\prime}\right) d r\right)^{1 / 2} \\
\leq & \|f\|_{L^{2}}, \\
L_{2} \leq & \left(\int_{2}^{\infty} r^{-d} d r\right)^{1 / 2} \\
& \times\left(\int_{2}^{\infty} r^{2 d-1-2 \gamma} r^{n-1} \int_{S^{n-1}}\left|\widehat{f}\left(r \xi^{\prime}\right)\right|^{2} d \sigma\left(\xi^{\prime}\right) d r\right)^{1 / 2} \\
\leq & \|f\|_{L_{d-\gamma-1 / 2}^{2}} \cdot
\end{aligned}
$$

Proposition 16 is proved.

Proof of Theorem 14. Denote

$$
\begin{aligned}
& m_{1}(t,|\xi|)=e^{-t} \cosh \left(t \sqrt{1-|\xi|^{2 \alpha}}\right) \\
& m_{2}(t,|\xi|)=e^{-t} \frac{\sinh \left(t \sqrt{1-|\xi|^{2 \alpha}}\right)}{\sqrt{1-|\xi|^{2 \alpha}}} .
\end{aligned}
$$

It is not hard to verify that

$$
\begin{aligned}
&\left|m_{1}(t,|\xi|)\right| \preceq 1, \quad\left|m_{2}(t,|\xi|)\right| \preceq(1+|\xi|)^{-\alpha}, \\
& \forall(t, \xi) \in \mathbb{R}^{+} \times \mathbb{R}^{n} .
\end{aligned}
$$

Since

$$
\begin{aligned}
u(t, x) & =m_{1}(t, D) f(x)+m_{2}(t, D) f(x)+m_{2}(t, D) g(x) \\
& =w(t, D) f(x)+m_{2}(t, D) g(x),
\end{aligned}
$$

Theorem 14 will be proved if we can show, as $t \rightarrow 0^{+}$,

$$
m_{2}(t, D) g(x) \longrightarrow 0, \quad \text { a.e. } x \in \mathbb{R}^{n}
$$

for $g \in L_{s-\alpha}^{2}\left(\mathbb{R}^{n}\right)$ and

$$
w(t, D) f(x) \longrightarrow f(x), \quad \text { a.e. } x \in \mathbb{R}^{n}
$$

for $f \in L_{s}^{2}\left(\mathbb{R}^{n}\right)$. The proof of the two limits is similar and we will only show the second convergence. Note that the above convergence always holds for Schwarz function $f$. So a further boundedness on the maximal function

$$
w^{*} f(x)=\sup _{t>0}|w(t, D) f(x)|
$$

that

$$
\left\|w^{*} f(x)\right\|_{L^{2}\left(d x /|x|^{d}\right)} \preceq\|f\|_{L_{s}^{2}\left(\mathbb{R}^{n}\right)}, \quad s>\frac{1}{2},
$$

is enough to imply Theorem 14 .

Next we will prove (181). By (176) and Proposition 16,

$$
\left\|m_{1}^{*} f(x)\right\|_{L^{2}\left(d x /|x|^{d}\right)} \preceq\|f\|_{L_{l}^{2}\left(\mathbb{R}^{n}\right)}, \quad \forall l>d-\frac{1}{2}
$$

Fix $s>1 / 2$. Taking $1<d<s+1 / 2$ and $l$ close to $d-1 / 2$, we have $l<s$ and thus

$$
\left\|m_{1}^{*} f(x)\right\|_{L^{2}\left(d x /|x|^{d}\right)} \preceq\|f\|_{L_{s}^{2}\left(\mathbb{R}^{n}\right)} .
$$

Applying Proposition 16 with $\gamma=\alpha$ and $1<d<1+2 \alpha$, we have

$$
\left\|m_{2}^{*} f(x)\right\|_{L^{2}\left(d x /|x|^{d}\right)} \preceq\|f\|_{\dot{L}_{1 / 2}^{2}\left(\mathbb{R}^{n}\right)} \leq\|f\|_{L_{s}^{2}\left(\mathbb{R}^{n}\right)} .
$$

Since

$$
w^{*} f(x) \leq m_{1}^{*} f(x)+m_{2}^{*} f(x)
$$

we proved (181) when $n \geq 2$ (note that Proposition 16 was proved only when $n \geq 2)$.

For $n=1$, instead of (181), we will show

$$
\left\|w^{*} f(x)\right\|_{L^{2}([-N, N])} \preceq N^{1 / 2}\|f\|_{L_{s}^{2}(\mathbb{R})}, \quad s>\frac{1}{2},
$$


which is also enough to obtain the pointwise convergence. Taking $h(x) \in L^{2}([-N, N])$, we have

$$
\begin{aligned}
& \int_{-N}^{N} w(t(x), D) f(x) h(x) d x \\
& =\int_{-N}^{N} \int_{R} w(t(x), \xi) \hat{f}(\xi) e^{i\langle x, \xi\rangle} d \xi h(x) d x \\
& =\int_{\mathbb{R}} \widehat{f}(\xi) \int_{-N}^{N} w(t(x), \xi) e^{i\langle x, \xi\rangle} h(x) d x d \xi \\
& \leq\left(\int_{\mathbb{R}}|\widehat{f}(\xi)|^{2}\left(1+|\xi|^{2}\right)^{s} d \xi\right)^{1 / 2} \\
& \quad \times\left(\int_{\mathbb{R}} \frac{\left|\int_{-N}^{N} w(t(x), \xi) e^{i\langle x, \xi\rangle} h(x) d x\right|^{2}}{\left(1+|\xi|^{2}\right)^{s}} d \xi\right)^{1 / 2} .
\end{aligned}
$$

Noting that

$$
w(t(x), \xi)=m_{1}(t(x), \xi)+m_{2}(t(x), \xi) \preceq 1, \quad \forall(x, \xi),
$$

we have

$$
\left|\int_{-N}^{N} w(t(x), \xi) e^{i\langle x, \xi\rangle} h(x) d x\right|^{2} \preceq N\|h\|_{L^{2}([-N, N])}^{2} .
$$

Therefore

$$
\int_{-N}^{N} w(t(x), D) f(x) h(x) d x \preceq N^{1 / 2}\|f\|_{L_{s}^{2}} \cdot\|h\|_{L^{2}([-N, N])}
$$

and by duality

$$
\|w(t(x), D) f(x)\|_{L^{2}([-N, N])} \leq N^{1 / 2}\|f\|_{L_{s}^{2}(\mathbb{R})}, \quad s>\frac{1}{2},
$$

from which (186) follows.

\section{Appendix}

We study the Cauchy problem

$$
\begin{array}{r}
u_{t t}(t, x)+2 b u_{t}(t, x)+(-\Delta)^{\alpha} u(t, x)=0, \\
(t, x) \in[0, \infty) \times \mathbb{R}^{n}, \\
u(0, x)=f(x), \quad u_{t}(0, x)=g(x) .
\end{array}
$$

We claim that the solution, in the Fourier transform side, is given by

$$
\begin{aligned}
\widehat{\mathcal{u}}(t, \xi)= & e^{-b t} \cosh \left(t \sqrt{b^{2}-|\xi|^{2 \alpha}}\right) \widehat{f}(\xi) \\
& +e^{-b t} \frac{\sinh \left(t \sqrt{b^{2}-|\xi|^{2 \alpha}}\right)}{\sqrt{b^{2}-|\xi|^{2 \alpha}}}(b \widehat{f}(\xi)+\widehat{g}(\xi)) .
\end{aligned}
$$

To verify this fact, we write the solution as

$$
u(t, x)=\Lambda(t, L) f(x)+\Gamma(t, L) g(x),
$$

where

$$
\begin{gathered}
\Gamma(t, L)=e^{-b t} \frac{\sinh (t \sqrt{L})}{\sqrt{L}}, \\
\Lambda(t, L)=b e^{-b t} \frac{\sinh (t \sqrt{L})}{\sqrt{L}}+e^{-b t} \cosh (t \sqrt{L}), \\
L=b^{2}-(-\Delta)^{\alpha} .
\end{gathered}
$$

Take derivative,

$$
\begin{aligned}
u_{t}= & -\left(b^{2} e^{-b t} \frac{\sinh (t \sqrt{L})}{\sqrt{L}}-b e^{-b t} \cosh (t \sqrt{L})\right) \\
& \times f-b e^{-b t} \cosh (t \sqrt{L}) f+e^{-b t} \sqrt{L} \sinh (t \sqrt{L}) f \\
& -b e^{-b t} \frac{\sinh (t \sqrt{L})}{\sqrt{L}} g+e^{-b t} \cosh (t \sqrt{L}) g \\
= & -b^{2} e^{-b t} \frac{\sinh (t \sqrt{L})}{\sqrt{L}} f+e^{-b t} \frac{b^{2}-(-\Delta)^{\alpha}}{\sqrt{L}} \sinh (t \sqrt{L}) f \\
& +e^{-b t} \cosh (t \sqrt{L}) g-b e^{-b t} \frac{\sinh (t \sqrt{L})}{\sqrt{L}} g .
\end{aligned}
$$

Thus

$$
\begin{aligned}
u_{t}= & e^{-b t} \frac{-(-\Delta)^{\alpha}}{\sqrt{L}} \sinh (t \sqrt{L}) f+e^{-b t} \cosh (t \sqrt{L}) g \\
& -b e^{-b t} \frac{\sinh (t \sqrt{L})}{\sqrt{L}} g, \\
u_{t t}= & b e^{-b t} \frac{(-\Delta)^{\alpha}}{\sqrt{L}} \sinh (t \sqrt{L}) f-b e^{-b t} \cosh (t \sqrt{L}) g \\
& +b^{2} e^{-b t} \frac{\sinh (t \sqrt{L})}{\sqrt{L}} g-e^{-b t}(-\Delta)^{\alpha} \cosh (t \sqrt{L}) f \\
& +e^{-b t} \sqrt{L} \sinh (t \sqrt{L}) g-b e^{-b t} \cosh (t \sqrt{L}) g .
\end{aligned}
$$

Therefore,

$$
\begin{aligned}
2 b e^{b t} u_{t}= & 2 b \frac{-(-\Delta)^{\alpha}}{\sqrt{L}} \sinh (t \sqrt{L}) f+2 b \cosh (t \sqrt{L}) g \\
& -2 b^{2} \frac{\sinh (t \sqrt{L})}{\sqrt{L}} g
\end{aligned}
$$




$$
\begin{aligned}
e^{b t} u_{t t}= & b \frac{(-\Delta)^{\alpha}}{\sqrt{L}} \sinh (t \sqrt{L}) f-2 b \cosh (t \sqrt{L}) g \\
& +b^{2} \frac{\sinh (t \sqrt{L})}{\sqrt{L}} g-(\Delta)^{\alpha} \cosh (t \sqrt{L}) f \\
& +\sqrt{L} \sinh (t \sqrt{L}) g .
\end{aligned}
$$

Thus,

$$
\begin{aligned}
e^{b t} u_{t t} & +2 b e^{b t} u_{t} \\
= & \frac{-(-\Delta)^{\alpha}}{\sqrt{L}} b \sinh (t \sqrt{L}) f-b^{2} \frac{\sinh (t \sqrt{L})}{\sqrt{L}} g \\
& -(-\Delta)^{\alpha} \cosh (t \sqrt{L}) f+\frac{b^{2}-(-\Delta)^{\alpha}}{\sqrt{L}} \sinh (t \sqrt{L}) g \\
= & \frac{-(-\Delta)^{\alpha}}{\sqrt{L}} b \sinh (t \sqrt{L}) f-(-\Delta)^{\alpha} \cosh (t \sqrt{L}) f \\
& -\frac{(-\Delta)^{\alpha}}{\sqrt{L}} \sinh (t \sqrt{L}) g \\
= & -(-\Delta)^{\alpha}\left\{\cosh (t \sqrt{L}) f+\frac{\sinh (t \sqrt{L})}{\sqrt{L}}(b f+g)\right\} .
\end{aligned}
$$

Thus,

$$
\begin{aligned}
u_{t t}+2 b u_{t}= & -(-\Delta)^{\alpha}(u) \\
= & -(-\Delta)^{\alpha}\left(\frac{b \sinh (t \sqrt{L})}{\sqrt{L}}+\cosh (t \sqrt{L})\right) f \\
& -(-\Delta)^{\alpha} \frac{\sinh (t \sqrt{L})}{\sqrt{L}} g .
\end{aligned}
$$

This shows the claim.

\section{Conflict of Interests}

The authors declare that there is no conflict of interests regarding the publication of this paper.

\section{Acknowledgment}

This work is partially supported by the NSF of China (Grant nos. 11271330 and 11201103).

\section{References}

[1] H. Bellout and A. Friedman, "Blow-up estimates for a nonlinear hyperbolic heat equation," SIAM Journal on Mathematical Analysis, vol. 20, no. 2, pp. 354-366, 1989.
[2] M. D. Blair, H. F. Smith, and C. D. Sogge, "On Strichartz estimates for Schrödinger operators in compact manifolds with boundary," Proceedings of the American Mathematical Society, vol. 136, no. 1, pp. 247-256, 2008.

[3] T. Cazenave, Semilinear Schrödinger Equations, vol. 10 of Courant Lecture Notes in Mathematics, New York University Courant Institute of Mathematical Sciences, New York, NY, USA, 2003.

[4] T. Cazenave and F. B. Weissler, "Rapidly decaying solutions of the nonlinear Schrödinger equation," Communications in Mathematical Physics, vol. 147, no. 1, pp. 75-100, 1992.

[5] J. Chen, D. Fan, and L. Sun, "Hardy space estimates for the wave equation on compact Lie groups," Journal of Functional Analysis, vol. 259, no. 12, pp. 3230-3264, 2010.

[6] Y. Giga, "Solutions for semilinear parabolic equations in $L^{p}$ and regularity of weak solutions of the Navier-Stokes system," Journal of Differential Equations, vol. 62, no. 2, pp. 186-212, 1986.

[7] M. Hieber and J. Prüss, "Heat kernels and maximal $L^{p}-L^{q}$ estimates for parabolic evolution equations," Communications in Partial Differential Equations, vol. 22, no. 9-10, pp. 1647-1669, 1997.

[8] M. Keel and T. Tao, "Endpoint Strichartz estimates," American Journal of Mathematics, vol. 120, no. 5, pp. 955-980, 1998.

[9] H. Lindblad and C. D. Sogge, "On existence and scattering with minimal regularity for semilinear wave equations," Journal of Functional Analysis, vol. 130, no. 2, pp. 357-426, 1995.

[10] B. Marshall, W. Strauss, and S. Wainger, " $L^{p}-L^{q}$ estimates for the Klein-Gordon equation," Journal de Mathématiques Pures et Appliquées, vol. 59, no. 4, pp. 417-440, 1980.

[11] C. Miao, B. Yuan, and B. Zhang, "Strong solutions to the nonlinear heat equation in homogeneous Besov spaces," Nonlinear Analysis: Theory, Methods \& Applications, vol. 67, no. 5, pp. 13291343, 2007.

[12] A. Miyachi, "On some Fourier multipliers for $H^{p}\left(\mathbb{R}^{n}\right)$," Journal of the Faculty of Science. University of Tokyo IA, vol. 27, no. 1, pp. 157-179, 1980.

[13] G. Mockenhaupt, A. Seeger, and C. D. Sogge, "Local smoothing of Fourier integral operators and Carleson-Sjölin estimates," Journal of the American Mathematical Society, vol. 6, no. 1, pp. 65-130, 1993.

[14] D. Müller and E. M. Stein, " $L^{p}$-estimates for the wave equation on the Heisenberg group," Revista Matemática Iberoamericana, vol. 15, no. 2, pp. 297-334, 1999.

[15] M. Nakao and K. Ono, "Existence of global solutions to the Cauchy problem for the semilinear dissipative wave equations," Mathematische Zeitschrift, vol. 214, no. 2, pp. 325-342, 1993.

[16] J. C. Peral, " $L^{p}$ estimates for the wave equation," Journal of Functional Analysis, vol. 36, no. 1, pp. 114-145, 1980.

[17] A. Seeger, C. D. Sogge, and E. M. Stein, "Regularity properties of Fourier integral operators," Annals of Mathematics, vol. 134, no. 2, pp. 231-251, 1991.

[18] G. Staffilani and D. Tataru, "Strichartz estimates for a Schrödinger operator with nonsmooth coefficients," Communications in Partial Differential Equations, vol. 27, no. 7-8, pp. 1337-1372, 2002.

[19] A. Stefanov, "Strichartz estimates for the Schrödinger equation with radial data," Proceedings of the American Mathematical Society, vol. 129, no. 5, pp. 1395-1401, 2001.

[20] E. Terraneo, "Non-uniqueness for a critical non-linear heat equation," Communications in Partial Differential Equations, vol. 27, no. 1-2, pp. 185-218, 2002. 
[21] G. Todorova and B. Yordanov, "Critical exponent for a nonlinear wave equation with damping," Journal of Differential Equations, vol. 174, no. 2, pp. 464-489, 2001.

[22] M. C. Vilela, "Inhomogeneous Strichartz estimates for the Schrödinger equation," Transactions of the American Mathematical Society, vol. 359, no. 5, pp. 2123-2136, 2007.

[23] F. B. Weissler, "Existence and nonexistence of global solutions for a semilinear heat equation," Israel Journal of Mathematics, vol. 38, no. 1-2, pp. 29-40, 1981.

[24] N. Hayashi, E. I. Kaikina, and P. I. Naumkin, "Damped wave equation with a critical nonlinearity," Transactions of the American Mathematical Society, vol. 358, no. 3, pp. 1165-1185, 2006.

[25] R. Ikehata and K. Tanizawa, "Global existence of solutions for semilinear damped wave equations in $\mathbb{R}^{N}$ with noncompactly supported initial data," Nonlinear Analysis: Theory, Methods \& Applications, vol. 61, no. 7, pp. 1189-1208, 2005.

[26] R. Ikehata, Y. Miyaoka, and T. Nakatake, "Decay estimates of solutions for dissipative wave equations in $\mathbb{R}^{N}$ with lower power nonlinearities," Journal of the Mathematical Society of Japan, vol. 56, no. 2, pp. 365-373, 2004.

[27] P. Marcati and K. Nishihara, “The $L^{p}-L^{q}$ estimates of solutions to one-dimensional damped wave equations and their application to the compressible flow through porous media," Journal of Differential Equations, vol. 191, no. 2, pp. 445-469, 2003.

[28] A. Matsumura, "On the asymptotic behavior of solutions of semi-linear wave equations," Publications of the Research Institute for Mathematical Sciences, vol. 12, no. 1, pp. 169-189, 1976.

[29] C. Miao, B. Yuan, and B. Zhang, "Well-posedness of the Cauchy problem for the fractional power dissipative equations," Nonlinear Analysis: Theory, Methods \& Applications, vol. 68, no. 3, pp. 461-484, 2008.

[30] C. Miao and B. Zhang, "The Cauchy problem for semilinear parabolic equations in Besov spaces," Houston Journal of Mathematics, vol. 30, no. 3, pp. 829-878, 2004.

[31] T. Narazaki, " $L^{p}-L^{q}$ estimates for damped wave equations and their applications to semi-linear problem," Journal of the Mathematical Society of Japan, vol. 56, no. 2, pp. 585-626, 2004.

[32] T. Narazaki, " $L^{p}-L^{q}$ estimates for damped wave equations with odd initial data," Electronic Journal of Differential Equations, vol. 2005, pp. 1-17, 2005.

[33] K. Nishihara, " $L^{p}-L^{q}$ estimates of solutions to the damped wave equation in 3-dimensional space and their application," Mathematische Zeitschrift, vol. 244, no. 3, pp. 631-649, 2003.

[34] H. Volkmer, "Asymptotic expansion of $L^{2}$-norms of solutions to the heat and dissipative wave equations," Asymptotic Analysis, vol. 67, no. 1-2, pp. 85-100, 2010.

[35] Q. S. Zhang, "A blow-up result for a nonlinear wave equation with damping: the critical case," Comptes Rendus de l'Académie des Sciences I, vol. 333, no. 2, pp. 109-114, 2001.

[36] J. Chen, Q. Deng, Y. Ding, and D. Fan, "Estimates on fractional power dissipative equations in function spaces," Nonlinear Analysis: Theory, Methods \& Applications, vol. 75, no. 5, pp. 2959-2974, 2012.

[37] Z. Zhai, "Strichartz type estimates for fractional heat equations," Journal of Mathematical Analysis and Applications, vol. 356, no. 2, pp. 642-658, 2009.

[38] A. Miyachi, "On some estimates for the wave equation in $L^{p}$ and $H^{p}$," Journal of the Faculty of Science. University of Tokyo IA, vol. 27, no. 2, pp. 331-354, 1980.
[39] A. Miyachi, "On some singular Fourier multipliers," Journal of the Faculty of Science. University of Tokyo IA, vol. 28, no. 2, pp. 267-315, 1981.

[40] K. M. Rogers, "A local smoothing estimate for the Schrödinger equation," Advances in Mathematics, vol. 219, no. 6, pp. 21052122, 2008.

[41] L. Carleson, "Some analytic problems related to statistical mechanics," in Euclidean Harmonic Analysis, vol. 779 of Lecture Notes in Mathematics, pp. 5-45, Springer, Berlin, Germany, 1980.

[42] S. Lee, "On pointwise convergence of the solutions to Schrödinger equations in $\mathbb{R}^{2}$," International Mathematics Research Notices, vol. 2006, Article ID 32597, 21 pages, 2006.

[43] P. Sjölin, "Regularity of solutions to the Schrödinger equation," Duke Mathematical Journal, vol. 55, no. 3, pp. 699-715, 1987.

[44] L. Vega, "Schrödinger equations: pointwise convergence to the initial data," Proceedings of the American Mathematical Society, vol. 102, no. 4, pp. 874-878, 1988.

[45] J. Bergh and J. Löfström, Interpolation Spaces. An Introduction, Grundlehren der Mathematischen Wissenschaften, no. 223, Springer, Berlin, Germany, 1976.

[46] H. Triebel, Theory of Function Spaces, vol. 78 of Monographs in Mathematics, Birkhäuser, Basel, Switzerland, 1983.

[47] A.-P. Calderón and A. Torchinsky, "Parabolic maximal functions associated with a distribution. II," Advances in Mathematics, vol. 24, no. 2, pp. 101-171, 1977.

[48] E. M. Stein, Harmonic Analysis: Real-Variable Methods, Orthogonality, and Oscillatory Integrals, vol. 43 of Princeton Mathematical Series, Princeton University Press, Princeton, NJ, USA, 1993.

[49] E. M. Stein and G. Weiss, Introduction to Fourier Analysis on Euclidean Spaces, Princeton Mathematical Series, no. 32, Princeton University Press, Princeton, NJ, USA, 1971.

[50] S. L. Wang, "On the weighted estimate of the solution associated with the Schrödinger equation," Proceedings of the American Mathematical Society, vol. 113, no. 1, pp. 87-92, 1991. 


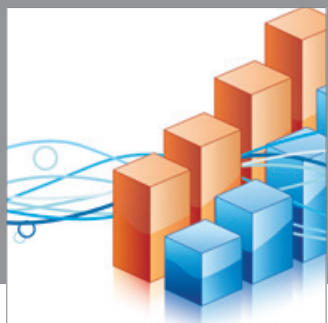

Advances in

Operations Research

mansans

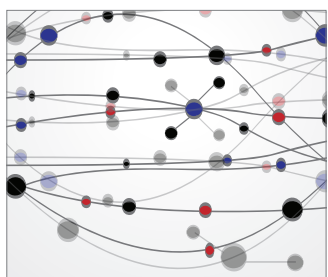

The Scientific World Journal
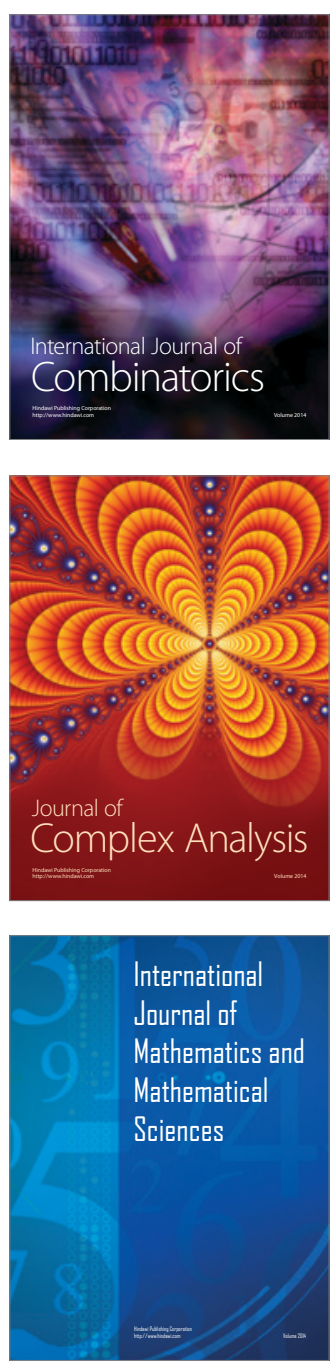
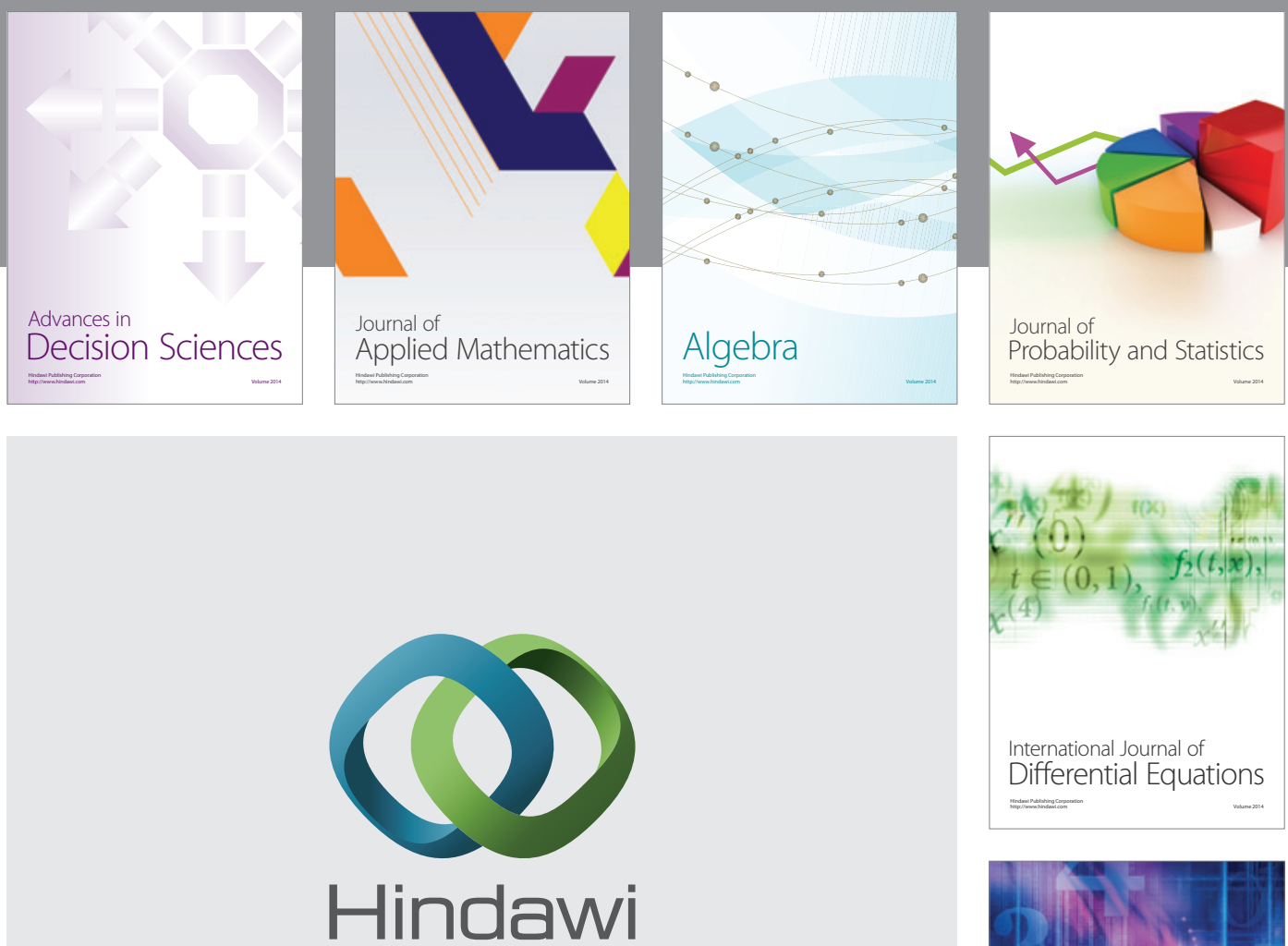

Submit your manuscripts at http://www.hindawi.com
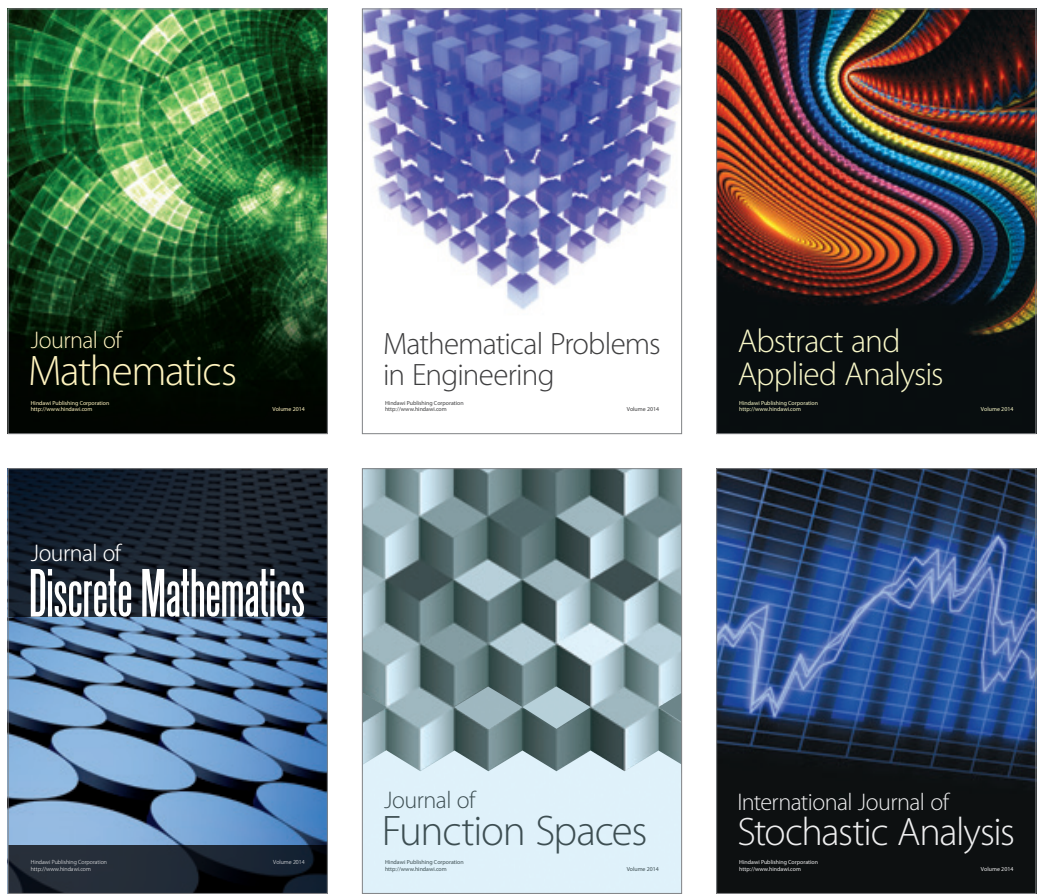

Journal of

Function Spaces

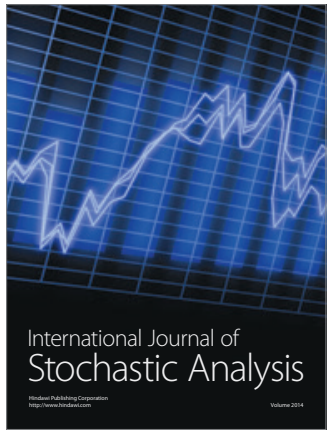

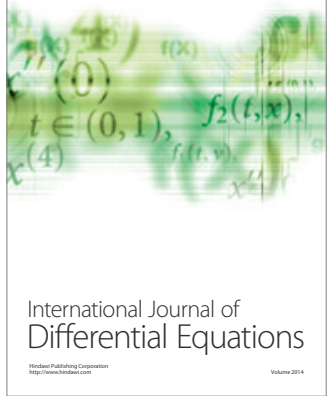
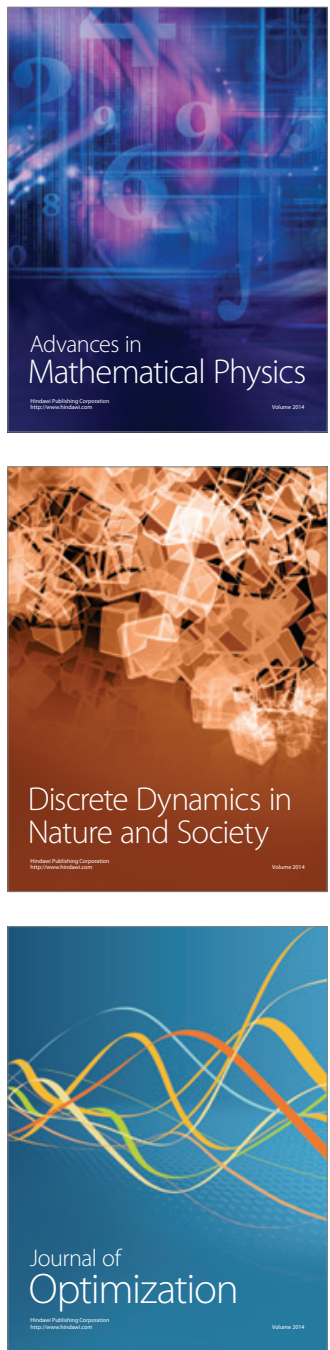